\title{
Augmin regulates kinetochore tension and spatial arrangement of spindle microtubules by nucleating bridging fibers
}

\author{
Martina Manenica $^{1 \#}$, Valentina Štimac ${ }^{1 \#}$, Isabella Koprivec ${ }^{1 \#}$, Juraj Simunić ${ }^{1}$ \\ Iva M. Tolić ${ }^{1 *}$
}

${ }^{1}$ Division of Molecular Biology, Ruđer Bošković Institute, Bijenička cesta 54, 10000 Zagreb, Croatia

${ }^{\#}$ These authors contributed equally to this work.

*Corresponding author: I.M.T. (tolic@irb.hr) 


\begin{abstract}
The mitotic spindle functions as a molecular micromachine that evenly distributes chromosomes into two daughter cells during cell division. Spindle microtubules in human cells are mainly nucleated at the centrosome and on the lateral surface of existing microtubules by the augmin complex. However, it is unknown how the augmin-mediated nucleation affects functionally distinct microtubule bundles and consequently the forces within the spindle. Here we show, by using siRNA depletion and CRISPR knock-out of the augmin complex subunits HAUS6 or HAUS8, that augmin is crucial for the nucleation of bridging microtubules, which laterally link sister kinetochore fibers. Augmin depletion resulted in a reduction in the number of microtubules within bridging fibers by around $80 \%$ and in kinetochore fibers by $40 \%$, suggesting that the bridging microtubules are mainly nucleated at the surface of present microtubules. In augmin-depleted cells, the interkinetochore distance decreased preferentially for kinetochores that lack a bridging fiber, independently of the thickness of their k-fibers, implying that augmin affects forces on kinetochores largely via bridging fibers. Without augmin the number of bridging fibers decreased, with the remaining ones mostly confined to the spindle periphery with an increased overlap length. A slower poleward flux of microtubules after augmin depletion is indicative of slower sliding within the bridging fiber. Our results demonstrate a critical role of augmin in the formation of bridging microtubules and proper architecture of the metaphase spindle, suggesting a model where sliding of augmin-nucleated bridging microtubules promotes poleward flux of k-fibers and thus tension on kinetochores.
\end{abstract}




\section{INTRODUCTION}

The mitotic spindle has a sophisticated architecture that enables it to accurately segregate the chromosomes during cell division. It consists of three major classes of microtubules: kinetochore microtubules that form k-fibers connecting chromosomes to the spindle pole through kinetochores, overlap microtubules that form antiparallel arrays in the central part of the spindle, and astral microtubules that extend from the spindle poles towards the cell cortex (McIntosh, 2016; Prosser and Pelletier, 2017). During metaphase and early anaphase, overlap microtubule bundles are laterally attached to a pair of sister k-fibers resembling a bridge between them, which is why they are called bridging fibers (Kajtez et al., 2016; Vukušić et al., 2017). These fibers balance the tension between sister kinetochores and maintain the curved shape of the metaphase spindle (Kajtez et al., 2016; Polak et al., 2017; Tolic, 2018; Tolic and Pavin, 2016).

Spindle microtubules in human somatic cells are predominantly generated by two nucleation mechanisms: centrosome-dependent and augmin-dependent nucleation (Kirschner and Mitchison, 1986; Pavin and Tolić, 2016; Petry, 2016; Prosser and Pelletier, 2017; Wu et al., 2008; Zhu et al., 2008). Centrosome-dependent nucleation was long thought to be predominant in spindle assembly, however, numerous studies revealed that a significant number of microtubules also arises from pre-existing microtubules, through augmin, an eightsubunit protein complex that serves as a recruiter of the $\gamma$-tubulin ring complex ( $\gamma$-TuRC) required for microtubule nucleation (David et al., 2019; Goshima et al., 2008; Kamasaki et al., 2013; Lawo et al., 2009; Song et al., 2018; Uehara et al., 2009). Augmin-nucleated microtubules grow at an angle of $0-30^{\circ}$ relative to the mother microtubule (Kamasaki et al., 2013; Petry et al., 2013; Verma and Maresca, 2019) and show a directional bias towards kinetochores, resulting in the preserved polarity of the spindle once the initial kinetochoremicrotubule attachments form (David et al., 2019; Kamasaki et al., 2013). Depletion of augmin complex in different cell types results in impaired k-fiber formation, reduced interkinetochore distance, loss of spindle bipolarity and stability, mitotic delay, and a higher incidence of aneuploidy and cytokinesis failure (Hayward et al., 2014; Uehara and Goshima, 2010; Uehara et al., 2009; Wu et al., 2008; Zhu et al., 2008). Of the eight subunits in the complex, the two directly interacting subunits HAUS6 (hDgt6/FAM29A) and HAUS8 (hDgt4/Hice1) have been most extensively studied because of their ability to interact with a $\gamma$ TuRC adapter protein NEDD1 and pre-existing microtubules, respectively (Song et al., 2018; Uehara et al., 2009). However, how augmin-dependent microtubule amplification affects 
functionally distinct microtubule bundles within the spindle and consequently the forces acting on kinetochores remained unclear.

To explore how augmin-dependent microtubule nucleation contributes to the formation of bridging and k-fibers and forces within the spindle, we depleted HAUS6 or HAUS8 in hTERT-RPE1 and HeLa cells by using siRNA or CRISPR knock-out technology. We show that the augmin complex plays a vital role in bridging fiber nucleation, thereby regulating forces and the three-dimensional structure of the metaphase spindle. Whereas augminmediated nucleation represents the primary nucleation pathway for bridging microtubules, $\mathrm{k}$ fiber microtubules are to a large extent nucleated also through other pathways, most likely at the centrosome. In augmin-depleted cells, the interkinetochore distance decreases preferentially at kinetochores without a bridging fiber, in a manner independent of the thickness of their k-fibers, suggesting an important role of the augmin-generated bridging microtubules in the regulation of interkinetochore tension. Augmin depletion leads to straighter outermost k-fibers and thus a diamond-like shape of the spindle, which is indicative of decreased compression within the k-fibers and demonstrates a role of augmin-generated bridging microtubules in the regulation of spindle forces and shape. Furthermore, without augmin microtubule poleward flux slows down, suggesting that the sliding of microtubules is reduced. We propose that augmin affects tension on kinetochores by generating bridging microtubules from the lateral sides of kinetochore microtubules. The bridging microtubules slide apart, thereby pushing the associated sister k-fibers apart, which promotes poleward flux and tension acting on sister kinetochores. 


\section{RESULTS}

\section{Bridging microtubules are predominantly generated through augmin-dependent nucleation}

To explore how the microtubules that make up the bridging fibers are nucleated, we consider two main possibilities: centrosomal and augmin-dependent nucleation (Fig. 1A). In the first scenario, future bridging microtubules are nucleated at the pole and subsequently make lateral contact to kinetochore microtubules of the same polarity and non-kinetochore microtubules of the opposite polarity extending from the other spindle pole. In the second scenario, previously formed k-fibers serve as an augmin-dependent nucleation platform from which bridging microtubules branch off. This scenario is supported by the recent electron tomography work on spindles in human cells showing that ends of non-kinetochore microtubules interact with the wall of kinetochore microtubules (O'Toole et al., 2020). Finally, the two modes of nucleation may coexist within the spindle.

To analyze the contribution of augmin-dependent microtubule nucleation to bridging fiber formation, we depleted HAUS6 or HAUS8 components of the augmin complex by siRNA in hTERT-RPE1 (hereafter referred to as RPE1) cells stably expressing CENP-A-GFP and Centrin1-GFP, stained with SiR-tubulin (Fig. 1B, Supplementary Video 1). Augmin depletion was confirmed using immunocytochemistry and Western blot analysis (Supplementary Fig. S1A-E) together with the previously observed phenotype of long, curved bundles (Wu et al., 2008; Zhu et al., 2008) (Supplementary Video 1).

We acquired profiles of tubulin signal intensity along the bundles from metaphase spindles and extracted the value of this signal at two locations: at the central region around the minimum of the tubulin signal and at the k-fiber region $1.5 \mu \mathrm{m}$ away from the central region (Fig. 1C and Methods). We interpret the former value as the signal of the bridging fiber, $I_{\mathrm{b}}$, and the latter as the combined signal of the k-fiber and bridging fiber, $I_{\mathrm{bk}}$, because the bridging microtubules from one spindle half extend along the k-fiber about $2 \mu \mathrm{m}$ into the other spindle half (Jagrić et al., 2020) (Fig. 1C, inset). The signal of the k-fiber is then calculated as $I_{\mathrm{k}}=I_{\mathrm{bk}}-I_{\mathrm{b}}$ (Kajtez et al., 2016). By using the resulting tubulin signal intensities (Fig. 1D), we first estimated the number of microtubules in the bridging fiber in untreated RPE1 cells. Electron tomography of spindles in RPE1 cells showed that k-fibers consist of $n_{\mathrm{k}}$ $=12.6 \pm 1.7$ microtubules (O'Toole et al., 2020) (all data are given as mean \pm s.e.m.). Thus, the bridging fiber consists of $n_{\mathrm{b}}=I_{\mathrm{b}} \times n_{\mathrm{k}} / I_{\mathrm{k}}=11.3 \pm 1.5$ microtubules. 
Remarkably, HAUS6 depletion resulted in $80 \pm 4 \%$ reduction of the bridging fiber signal intensity and $39 \pm 5 \%$ reduction of the k-fiber signal intensity (Fig. 1D, E), indicating that augmin depletion affects not only k-fibers, but even more so bridging fibers. Based on these measurements, we estimate that after HAUS6 depletion bridging fibers consist of $2.3 \pm 0.6$ microtubules and k-fibers of $7.7 \pm 0.9$ microtubules, which we interpret as microtubules nucleated in an augmin-independent manner. Thus, $9.0 \pm 1.6$ microtubules in the bridging fiber and $4.9 \pm 1.2$ microtubules in the k-fiber are nucleated in an augmin-dependent manner.

Similarly, HAUS8 depletion resulted in strong reduction of bridging fibers (Fig. 1D, E), though the exact values were somewhat different from HAUS6 depletion possibly due to differences in siRNA efficiencies (Supplementary Fig. S1A-E). Moreover, $26 \pm 6 \%$ and $15 \pm$ $5 \%$ of all kinetochore pairs in HAUS6 and HAUS8 depleted cells, respectively, had no detectable bridging fibers, defined as those with the tubulin signal below the background signal (Fig. 1F; see Methods). These results reveal a crucial role of the augmin complex in the assembly of bridging fibers.

As a control for the method used to analyze tubulin signal intensities, we performed another type of analysis in which tubulin signal intensity of the bridging fiber was measured in a small square region at the bridging fiber center, and the signal intensity of the k-fiber in a region of the same size next to the kinetochore (Supplementary Fig. S1F and Methods). In untreated cells, this method yields $8.5 \pm 1.6$ microtubules in the bridging fiber, which is comparable to the value obtained from the profiles of tubulin signal intensity in Fig. $1 \mathrm{C}(\mathrm{p}=0.2)$. Moreover, this analysis confirmed the result that the bridging fibers were impaired to a larger extent than $\mathrm{k}$-fibers after augmin depletion $(66 \pm 6 \%$ and $59 \pm 8 \%$ decrease of the bridging fiber signal intensity and $39 \pm 7 \%$ and $26 \pm 9 \%$ decrease of the k-fiber signal after HAUS6 and HAUS 8 depletion, respectively; Supplementary Fig. S1F).

As a control for SiR-tubulin labeling, we examined HeLa cells stably expressing GFP- $\alpha$ tubulin in which microtubules were also stained by SiR-tubulin. The signal intensity profiles of the two tubulin markers acquired along individual bundles were similar (Supplementary Fig. S1G), suggesting that SiR-tubulin is a suitable marker for the study of microtubule bundles in the spindle. Taken together, these results suggest that the augmin-based nucleation is a major contributor to the formation of bridging fibers, whereas its contribution to the formation of $\mathrm{k}$-fibers is significant but less prominent. 


\section{A}
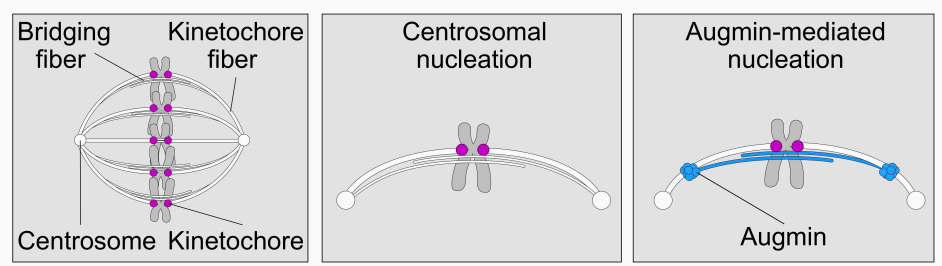

B
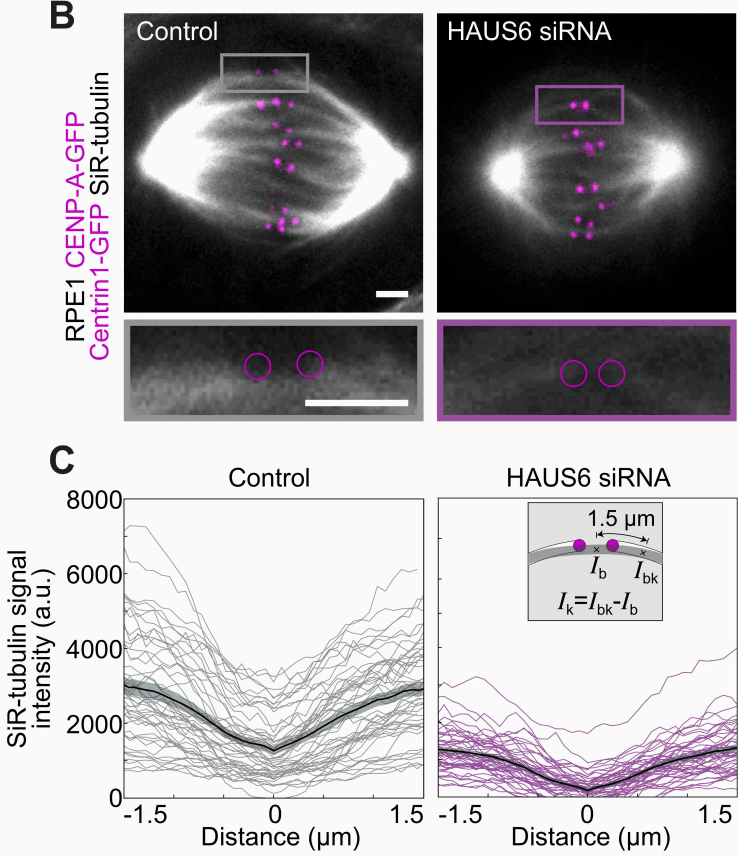

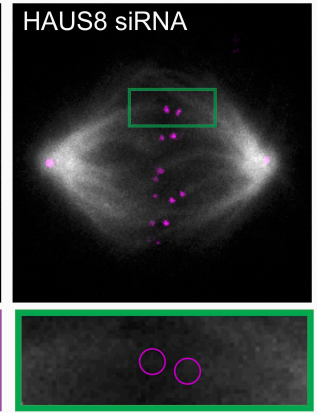

HAUS8 SiRNA

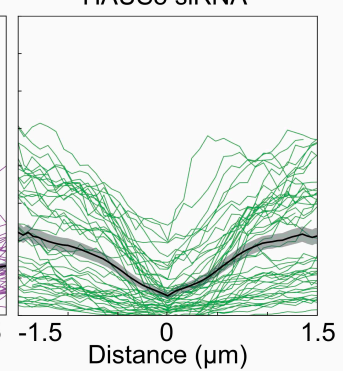

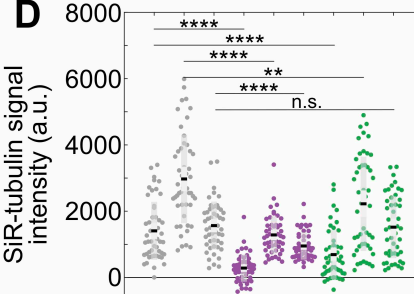

$I_{\mathrm{b}} I_{\mathrm{bk}} I_{\mathrm{k}} I_{\mathrm{b}} I_{\mathrm{bk}} I_{\mathrm{k}} I_{\mathrm{b}} I_{\mathrm{bk}} I_{\mathrm{k}}$

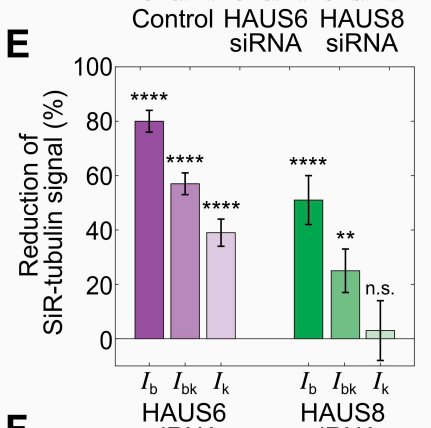

$\mathbf{F}$

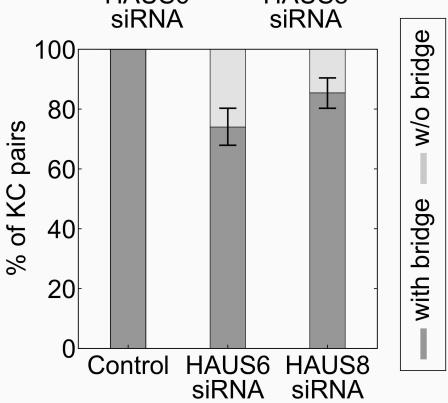

Figure 1. Augmin is crucial for the nucleation of bridging microtubules. (A) Schematic representation of the mitotic spindle in metaphase (left) and two pathways of bridging fiber formation: centrosomal nucleation (middle) and augmin-mediated nucleation (right). (B) Live images (single z-plane) of SiR-tubulin (gray) stained metaphase spindles in RPE1 cells stably expressing CENP-A-GFP and Centrin1-GFP (both in magenta) in control cells (left) and after HAUS6 (middle) or HAUS8 (right) depletion. Enlarged boxes show bridging fibers affected by HAUS6 or HAUS8 depletion compared to a bridging fiber in control cell. The positions of kinetochores are marked with magenta circles. Scale bars, $2 \mu \mathrm{m}$. (C) Intensity profiles of SiR-tubulin signal along the bundles in control cells (gray) and after HAUS6 (magenta) or HAUS8 (green) depletion. The center of the bridging fiber is set at a distance zero and the minimum intensity of the central part of the spindle was subtracted from the values of intensity profiles. $\mathrm{N}=10$ cells and 50 bundles for control and HAUS6 siRNAtreated cells, $\mathrm{N}=10$ cells and 48 bundles for HAUS8 siRNA-treated cells. Mean \pm SEM (thick black line and shaded area, respectively). The method for measuring the tubulin intensity of the bridging fiber and the k-fiber is depicted in the inset; the average of two k-fibers was calculated whenever both k-fibers resided in the same-z plane. (D) Univariate scatter plot of the tubulin signal intensities of bridging and k-fibers in control cells (gray) and upon HAUS6 (magenta) and HAUS8 (green) depletion. Boxes represent standard deviation (dark gray), 95\% confidence interval of the mean (light gray) and mean value (black). (E) Reduction of the tubulin signal in the bridging fiber $\left(I_{\mathrm{b}}\right)$, bundle consisting of the k-fiber and bridging fiber $\left(I_{\mathrm{bk}}\right)$, and the k-fiber alone $\left(I_{\mathrm{k}}\right)$ following HAUS6 (magenta) or HAUS8 (green) depletion, values are shown as mean \pm SEM. P-values were calculated using the absolute values of tubulin signal intensity of bridging or k-fibers following HAUS6 or HAUS8 
depletion, compared to the absolute values of tubulin signal intensity of corresponding fibers in control cells. (F) Fractions of kinetochore pairs with bridging fibers (dark gray) and with no distinguishable bridging fibers (light gray), values are shown as mean \pm SEM. All results were obtained from three independent experiments. Statistical analysis (D and E); t-test, p-value legend: $<0.0001(* * * *), 0.0001$ to $0.001(* * *), 0.001$ to $0.01(* *)$, 0.01 to $0.05(*), \geq 0.05(\mathrm{~ns})$.

\section{The interkinetochore distance decreases after augmin depletion preferentially at kinetochores with weaker bridging fibers}

The interkinetochore distance, which is a readout of interkinetochore tension (Waters et al., 1996), decreases after augmin depletion (Uehara et al., 2009; Zhu et al., 2008). Our measurements on RPE1 and HeLa cells also showed a reduced interkinetochore distance in augmin-depleted cells (Supplementary Fig. S2A-S2D). This reduction of interkinetochore tension may be due to weaker k-fibers (Uehara et al., 2009; Zhu et al., 2008). However, our finding that augmin predominantly nucleates bridging microtubules (Fig. 1) prompted us to hypothesize that the decrease in tension after augmin depletion may also result from weaker bridging fibers.

To test to what extent the observed reduction in interkinetochore distance can be related to the perturbation of bridging fibers in augmin-depleted cells, we divided kinetochore pairs into two groups: (1) those with a bridging fiber (i.e., signal intensity of the bridging fiber $>500$ a.u., which corresponds to $\sim 30 \%$ of the bridging fiber intensity in control cells), and (2) those with significantly reduced or undetectable signal intensities at the expected locations of bridging fibers (<500 a.u.), which we for simplicity refer to as kinetochore pairs without bridging fibers (Fig. 2A, Supplementary Video 2). Remarkably, kinetochore pairs without bridging fibers had a significantly shorter interkinetochore distance than kinetochore pairs with bridging fibers (Fig. 2B). The interkinetochore distance of kinetochores without a bridging fiber was $0.76 \pm 0.01 \mu \mathrm{m}(\mathrm{N}>40$ kinetochore pairs from 10 cells $)$ for HAUS6 and $0.70 \pm 0.02 \mu \mathrm{m}(\mathrm{N}>40$ kinetochore pairs from 10 cells $)$ for HAUS8 siRNA treated cells. In contrast, kinetochores with a bridging fiber had a distance of $0.83 \pm 0.02 \mu \mathrm{m}(\mathrm{N}>40$ kinetochore pairs from 10 cells $)$ and $0.88 \pm 0.01 \mu \mathrm{m}(\mathrm{N}>20$ kinetochore pairs from 10 cells $)$ for HAUS6 and HAUS8 depleted cells, respectively (Fig. 2B). While the value of the interkinetochore distance depended on the presence or absence of the bridging fiber, it did not correlate with k-fiber intensity within each group (Fig. 2C). Thus, bridging fibers play a role in the regulation of interkinetochore tension in a manner independent of k-fibers. 
Next, we explored the spatial distribution of kinetochore pairs with and without bridging fibers. In the inner part of the spindle, less than $2 \mu \mathrm{m}$ away from the spindle axis, kinetochore pairs with bridging fibers represented only $32 \pm 6 \%$ and $14 \pm 4 \%$ of all kinetochore pairs in HAUS6 and HAUS8 depleted cells, respectively (Fig. 2D). The situation was reversed in the outer part of the spindle, more than $2 \mu \mathrm{m}$ away from the spindle axis, where kinetochore pairs with bridging fibers represented $64 \pm 6 \%$ and $65 \pm 7 \%$ of all kinetochore pairs following the same treatments (Fig. 2D). To exclude the possibility that this result is affected by the analysis based only on those kinetochores that were well isolated from the neighboring microtubule bundles and thus suitable for tubulin signal measurements, we explored how the interkinetochore distance changes depending on the position of the kinetochore pair within the spindle, for all kinetochores. We found that kinetochore pairs in the outer part of the spindle of cells treated with HAUS6 or HAUS8 siRNA had a significantly larger interkinetochore distance than those in the inner part of the spindle (Fig. 2E), which coincides with predominant presence of bridging fibers on the outside (Fig. 2D). These results further support our conclusion that augmin has an important role in regulating interkinetochore tension through the nucleation of bridging microtubules. 
A

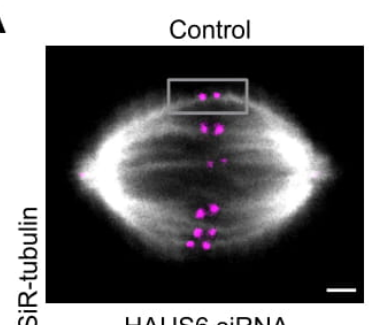

HAUS6 SiRNA

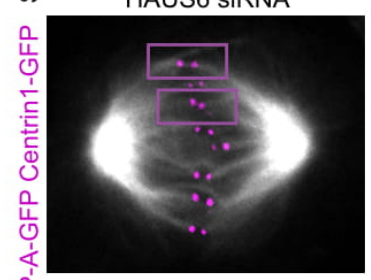

HAUS8 SiRNA

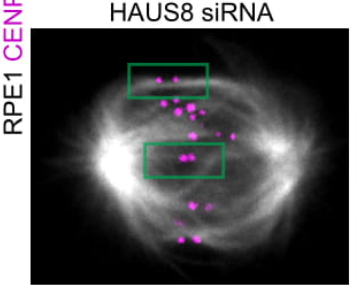

D

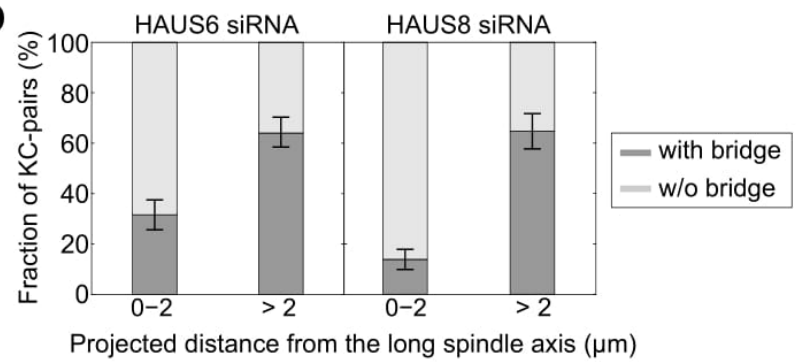

KCs with bridge

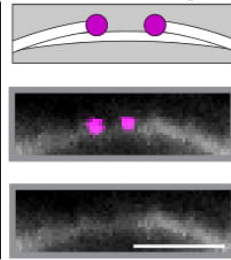

$\mathrm{KCs}$ with bridge
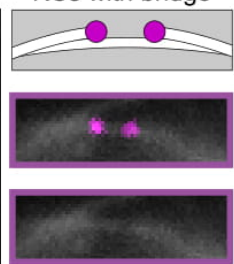

$\mathrm{KCs}$ with bridge
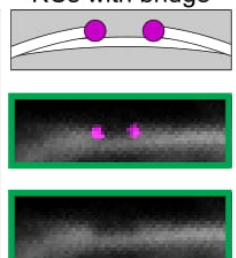
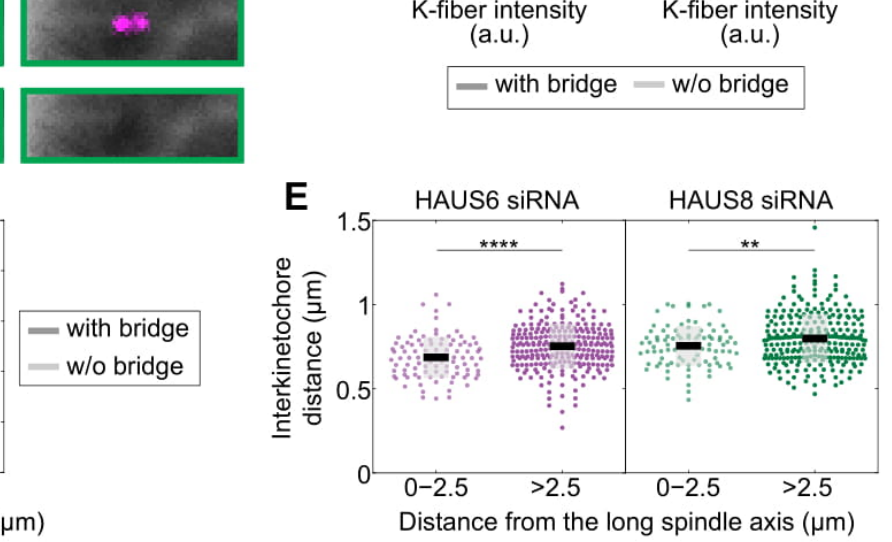

Figure 2. Shortening of the interkinetochore distance due to augmin depletion is related to bridging fiber reduction. (A) Live images (single z-plane) of metaphase spindles in RPE1 cells stably expressing CENP-AGFP and Centrin1-GFP (both in magenta) and stained with SiR-tubulin (gray). Enlarged boxes show kinetochore (KC) pairs with or without a bridging fiber (see text for definition). Gray frames denote control cells, magenta frames HAUS6 and green frames HAUS8 depleted cells. Brightness and contrast were adjusted for clarity. Scale bars, $2 \mu \mathrm{m}$. (B) Univariate scatter plot of the interkinetochore distance in HAUS6 (left) and HAUS8 (right) depleted cells ( $n=10$ cells, $n>50$ kinetochore pairs). Kinetochore pairs are divided into two groups: with bridging fiber (dark gray) and without bridging fiber (light gray). (C) Correlation of the interkinetochore distance and kfiber intensity for kinetochore pairs with (dark gray) and without (light gray) bridging fiber in HAUS6 (left) and HAUS8 (right) siRNA treated cells. Data represent a subset from B, in which only kinetochore pairs with kfibers well isolated from neighboring microtubules were taken into account. The interkinetochore distance values for kinetochore pairs with and without bridging fibers were $0.83 \pm 0.02$ and $0.76 \pm 0.02 \mu \mathrm{m}$ for HAUS6 and $0.89 \pm 0.02$ and $0.73 \pm 0.01 \mu \mathrm{m}$ for HAUS8 siRNA treated cells, respectively, in agreement with results from panel B. rs, Spearman correlation coefficient. (D) Fractions of kinetochore pairs with bridging fibers (dark gray) and without bridging fibers (light gray) depending on the projected distance from the long (pole-to-pole) spindle axis in HAUS6 (left) and HAUS8 (right) depleted cells. Fractions are given as mean \pm SEM. (E) Univariate scatter plot of the interkinetochore distance in HAUS6 (magenta) or HAUS8 (green) depleted cells depending on the 3D-distance from the long spindle axis. (B and E) Boxes represent standard deviation (dark gray), 95\% 
confidence interval of the mean (light gray) and mean value (black). All results were obtained from three independent experiments. Statistical analysis (B,C and E); t-test, p-value legend: $<0.0001(* * * *), 0.0001$ to $0.001(* * *), 0.001$ to $0.01(* *), 0.01$ to $0.05(*), \geq 0.05(\mathrm{~ns})$.

\section{Augmin-depleted spindles contain fewer overlap bundles, which have longer overlap regions and are located at the spindle periphery}

Our finding that the interkinetochore distance and bridging fibers were perturbed more in the inner part of the spindle after augmin depletion prompted us to examine the spatial distribution of bridging fibers and their overlap regions throughout the spindle. We used protein regulator of cytokinesis 1 (PRC1) as a marker because it preferentially crosslinks overlap microtubules (Li et al., 2018; Mollinari et al., 2002), thus providing a specific label for bridging fibers in metaphase spindles (Polak et al., 2017). We analyzed the metaphase spindles by taking a standard "side view" of the spindle and by rotating the 3D image stack of the spindle into an "end-on" view (Fig. 3A) (Novak et al., 2018). HeLa cells stably expressing PRC1-GFP and stained with SiR-DNA after treatment with HAUS6 or HAUS8 siRNA (Fig. 3B, Supplementary Video 3) were used to measure the number of PRC1-GFP bundles, their size and overlap lengths.

We found that the number of PRC1-labeled overlap bundles measured in an end-on view of spindles was almost halved; there were $20 \pm 2$ distinct bundles in HAUS6 and $22 \pm 1$ in HAUS8 siRNA treated cells, compared to $37 \pm 2$ distinct bundles in control cells (Fig. 3C). The analysis of side-view spindle images revealed that the antiparallel overlap regions in HAUS6 and HAUS8 siRNA treated cells were $8.7 \pm 0.3 \mu \mathrm{m}$ and $7.2 \pm 0.3 \mu \mathrm{m}$ long, respectively ( $\mathrm{n}=50$ overlaps from 10 cells in each case; Fig. 3D). This was roughly twice the length of overlaps in control cells, which were $4.3 \pm 0.1 \mu \mathrm{m}$ long ( $\mathrm{n}=50$ overlaps from 10 cells; Fig. 3D), consistent with previous measurements (Kajtez et al., 2016; Polak et al., 2017). The observed increase in overlap length was not proportional to the increase of spindle length, as overlaps constituted $39 \pm 1 \%, 69 \pm 4 \%$ and $61 \pm 4 \%$ of the spindle length in control cells, HAUS6, and HAUS8 siRNA treated cells, respectively (Supplementary Fig. S3A). Measurements of total PRC1 signal intensity in the spindle revealed that there was no statistically significant difference between control and HAUS6 $(14 \pm 19 \%$ reduction, $\mathrm{p}=0.50)$ or HAUS8 $(6 \pm 13 \%$ reduction, $\mathrm{p}=0.67)$ siRNA treated cells, indicating that there was no decrease, but only a redistribution of PRC1 within the spindle from a large number of short overlaps to a small number of long overlaps. Interestingly, the long curved bundles 
characteristic for augmin depletion (Goshima et al., 2008; Uehara et al., 2016; Wu et al., 2008; Zhu et al., 2008) exhibited PRC1 signal along most of their length, suggesting that they consist of antiparallel microtubules, even though contrary to bridging fibers, they form away from the kinetochore fibers and kinetochores (Supplementary Video 1 and 2). These results were corroborated by PRC1-antibody staining in unlabeled HeLa cells, which also showed a reduced number of overlaps and elongated PRC1 signal along the curved outer bundles after augmin depletion (Supplementary Fig. S3B-S3D), confirming that the observed effects are not a result of PRC1 overexpression. Thus, without augmin, the spindles contain fewer bridging fibers, which have longer overlaps.

The augmin-depleted cells showed a specific barrel-like distribution of the PRC1-GFP labeled bundles, with more overlap bundles being present around the perimeter of the spindle and fewer in the central part (Fig. 3B "end-on view" and 3E). However, staining with SiR-DNA showed a uniform distribution of DNA throughout the spindle cross-section, both in treated and control cells (Fig. 3B "end-on view"). In agreement with this result, kinetochores and tubulin signal were found uniformly distributed over the spindle cross-section in augmindepleted and SiR-tubulin stained RPE1 cells stably expressing CENP-A-GFP and Centrin1GFP (Fig. 3B "end-on view" of RPE1 cells). This observation indicates that k-fibers are present and roughly uniformly distributed throughout the spindle cross-section and is in agreement with our finding that augmin primarily affects bridging fibers, while k-fibers are less perturbed (Fig. 1).

To explore the role of augmin in defining the overall spindle geometry, we measured spindle length, spindle width as the distance between the outermost microtubule bundles, and the diameter of the metaphase plate as the distance between the outermost kinetochore pairs or the outermost chromosome regions in RPE1 and HeLa cells, respectively (see Methods and schemes in Fig. 4A and C). Spindles of both cell lines were wider in diameter (Fig. 4B and D, Table S1). Augmin-depleted spindles of HeLa cells were also longer, consistent with previous observations on Drosophila S2 cells and Xenopus egg extracts (Goshima et al., 2007; Petry et al., 2011). Yet, spindles in RPE1 cells shortened (Fig. 4B and D, Table S1), likely due to differences between cell lines. Interestingly, there was no difference in the diameter of the metaphase plate between control and augmin-depleted cells (Fig. 4B and D, Table S1). Overall, augmin depletion led to the impairment of bridging fibers, which in turn impaired the entire architecture of the metaphase spindle. 

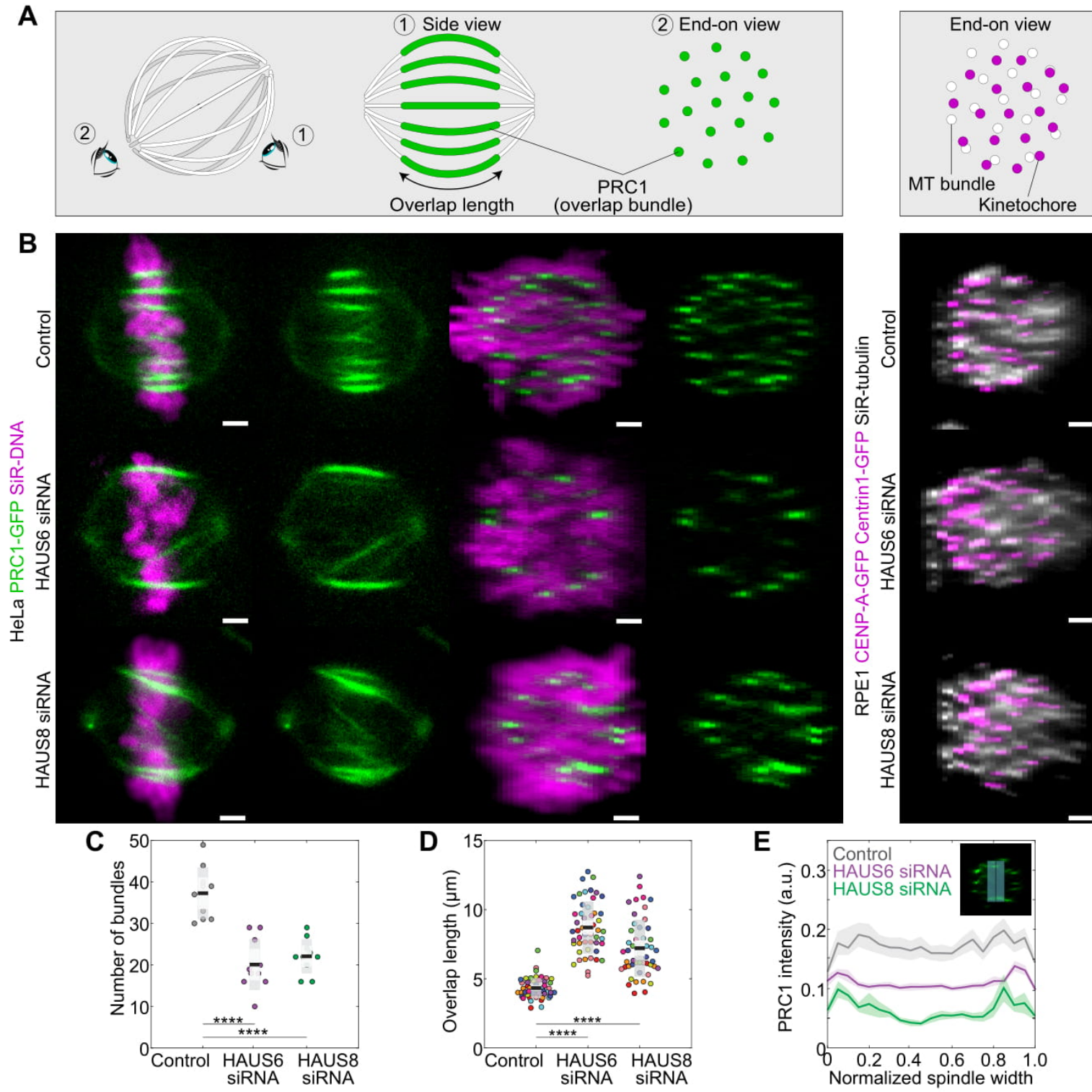

Overlap length

(overlap bundle)
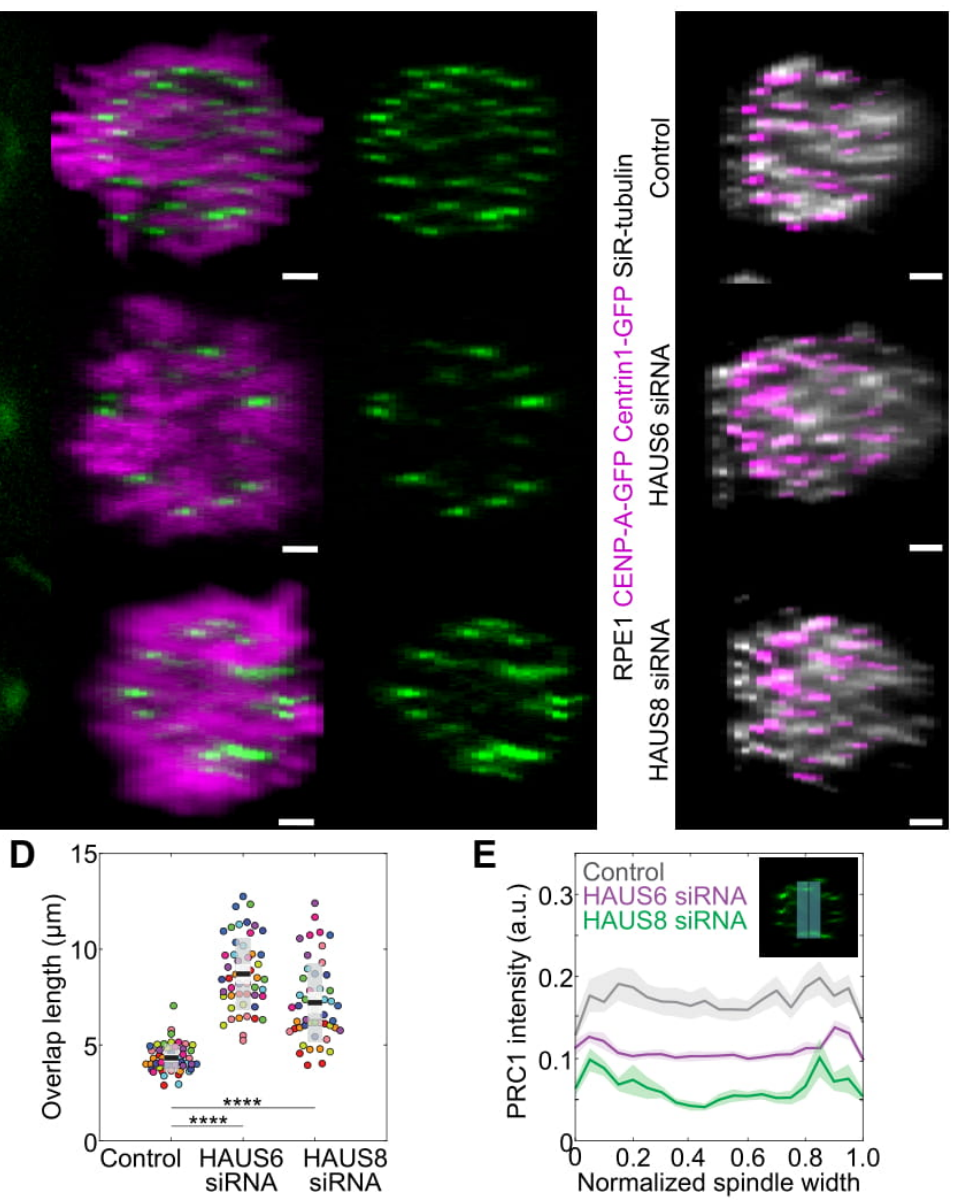

Figure 3. In augmin-depleted spindles the number of bridging fibers decreases, with the remaining ones having an increased overlap length and being located at the spindle periphery. (A) Schematic representations of different views of the spindle. Eye signs mark the angle for the side view (1) and the end-on view (2). Side view was used to measure the length of overlap regions (green) and end-on view to determine the number of bundles (green dots). Scheme of the end-on view of RPE1 cells stably expressing CENP-A-GFP and Centrin1-GFP and stained with SiR-tubulin (right). (B) Four columns on the left represent live images of metaphase spindles in HeLa cells stably expressing PRC1-GFP (green) and stained with SiR-DNA (magenta) in control (top) and after HAUS6 (middle) or HAUS8 (bottom) depletion. 1st and 2nd column: side view of the spindle; 3rd and 4th column: end-on view of the same spindle, showing a barrel-like arrangement of PRC1labeled bundles after augmin depletion. Images on the right show the end-on view of RPE1 cells stably expressing CENP-A-GFP and Centrin1-GFP (both in magenta) and stained with SiR-tubulin (gray) in control cells (top) and after HAUS6 (middle) and HAUS8 depletion (bottom). Side views are sum projections of 5 
bioRxiv preprint doi: https://doi.org/10.1101/2020.0910.291740; this version posted November 19, 2020. The copyright holder for this preprint (which was not certified by peer review) is the author/funder, who has granted bioRxiv a license to display the preprint in perpetuity. It is made available under aCC-BY-NC-ND 4.0 International license.

central z-slices $(\Delta \mathrm{z}=0.5 \mu \mathrm{m})$ and end-on views are sum projections of 10 central $\mathrm{z}$-slices $(\Delta \mathrm{z}=0.083 \mu \mathrm{m})$. Brightness and contrast were adjusted for clarity. Scale bars, $2 \mu \mathrm{m}$. (C) Univariate scatter plot of the number of bundles counted in the end-on view of the spindle. (D) Overlap length measured in the side view of the spindle. Each color in the plot corresponds to data obtained in one cell ( $n=10$ cells and $n=50$ overlaps). (C and D) Boxes represent standard deviation (dark gray), 95\% confidence interval of the mean (light gray) and mean value (black). (E) PRC1-GFP intensity profile measured in the end-on view of the spindle in control cells (gray) and after HAUS6 (magenta) and HAUS8 (green) depletion. The blue line in the inset marks the measured region (width: $2.5 \mu \mathrm{m}$ ). Mean (thick lines) and SEM (shaded areas). All results were obtained from three independent experiments. Statistical analysis (C and D); t-test, p-value legend: $<0.0001(* * * *), 0.0001$ to $0.001(* * *), 0.001$ to $0.01(* *), 0.01$ to $0.05(*), \geq 0.05(\mathrm{~ns})$.

A
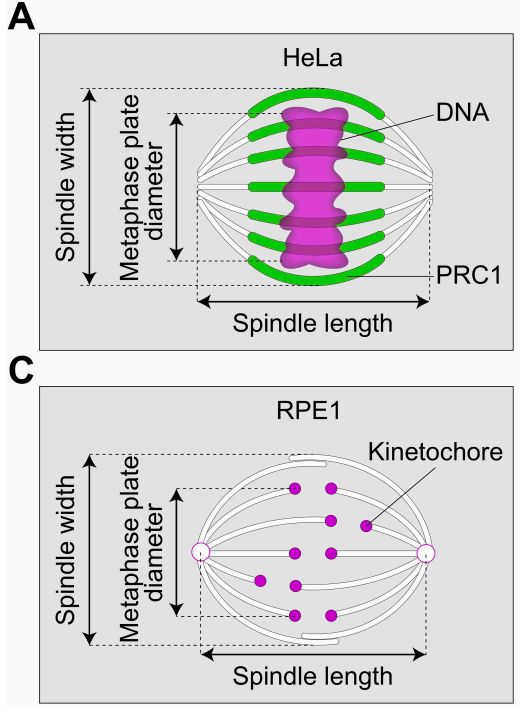

B
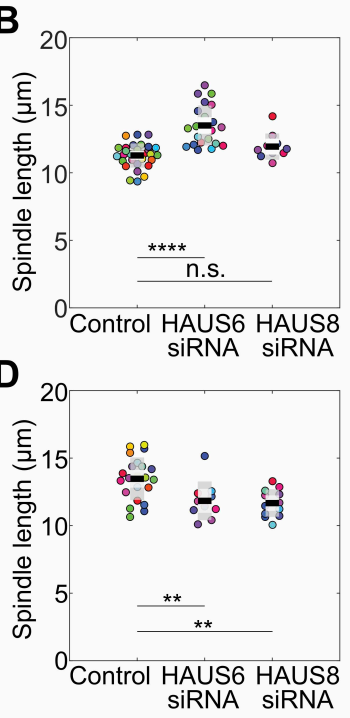
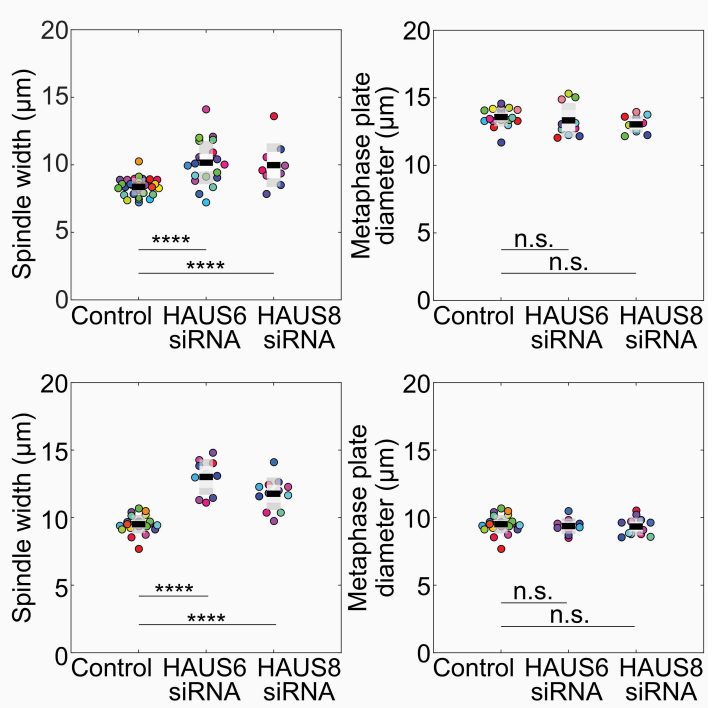

Figure 4. Spindle length and width, but not the diameter of the metaphase plate, change upon augmin depletion. (A) and (C) Measurements of spindle geometry in HeLa cells stably expressing PRC1-GFP (green) and stained with SiR-DNA (magenta) (A) and RPE1 cells stably expressing CENP-A-GFP and Centrin1-GFP (both in magenta) and stained with SiR-tubulin (white). (B and D) Univariate scatter plots of the spindle length (left), width (middle) and diameter of the metaphase plate (right). Each color in the plot corresponds to data obtained in one cell ( $\mathrm{n}=10$ cells). Boxes represent standard deviation (dark gray), 95\% confidence interval of the mean (light gray) and mean value (black). All results were obtained from three independent experiments. Statistical analysis (B and D); t-test, p-value legend: <0.0001 (****), 0.0001 to $0.001(* * *), 0.001$ to $0.01(* *)$, 0.01 to $0.05(*), \geq 0.05$ (ns). 


\section{Augmin depletion leads to straighter k-fibers and excessively long bundles on the spindle periphery}

Augmin-depleted spindles represent a unique system where three types of interactions between k-fibers and bridging fibers can be found within the same spindle: (1) k-fibers attached to bridging fibers, (2) k-fibers without a bridging fiber, and (3) solitary interpolar bundles without associated kinetochores. This is in contrast with control cells, where the first group dominates and the other two groups are rarely found (Polak et al., 2017). To gain insight into the contribution of each of these functionally distinct microtubule bundles to the maintenance of spindle geometry, we explored bundle shapes in HAUS8 siRNA treated RPE1 cells (Fig. 5A) and in RPE1 cells with induced CRISPR/Cas9 knock-out of HAUS8 (Fig. 5B, Supplementary Video 5). We traced the outermost bundles for each bundle type and compared them with each other as well as with the outermost bundles in control cells (Fig. $5 \mathrm{C})$.

The bundles without kinetochores had the longest contour length, followed by the outermost bundles in control cells, k-fibers with a bridging fiber, and finally, k-fibers without a bridging fiber (Fig. 5D and Table 1). To determine the curvature of the bundles, we fitted a circle to the bundle outline (Supplementary Fig. S4A), and found a drastic reduction of curvature for the k-fibers without bridging fibers in comparison with all other bundle types (Fig. 5E and Table 1). Similar results were obtained in RPE1 and HeLa cell lines treated with HAUS6 siRNA (Supplementary Fig. S4B-S4E and Table 1). Overall, the outer interpolar bundles without associated kinetochores are excessively long and make the spindle wider, whereas k-fibers lacking a bridging fiber are overly straight, ultimately resulting in a diamond-like shape of the spindle. This change in spindle shape is consistent with the prediction of our theoretical model (Kajtez et al., 2016). 
bioRxiv preprint doi: https://doi.org/10.1101/2020.09.10.291740; this version posted November 19, 2020. The copyright holder for this preprint (which was not certified by peer review) is the author/funder, who has granted bioRxiv a license to display the preprint in perpetuity. It is made available under aCC-BY-NC-ND 4.0 International license.

A

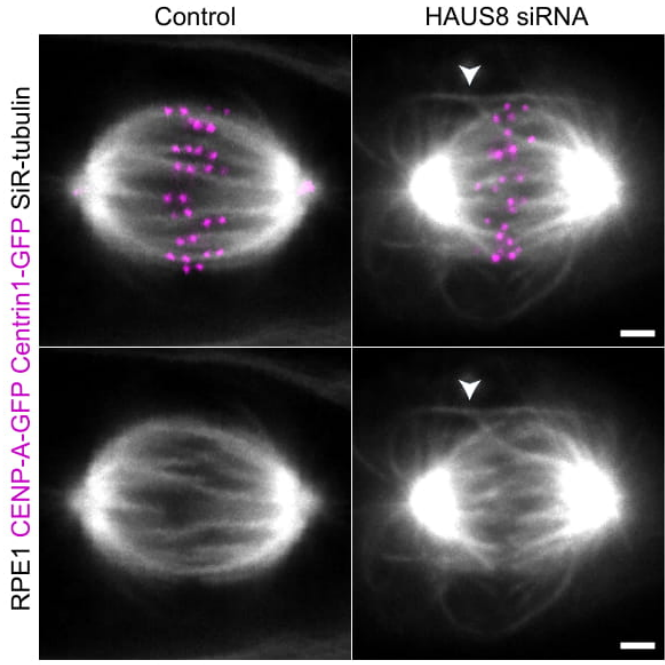

B

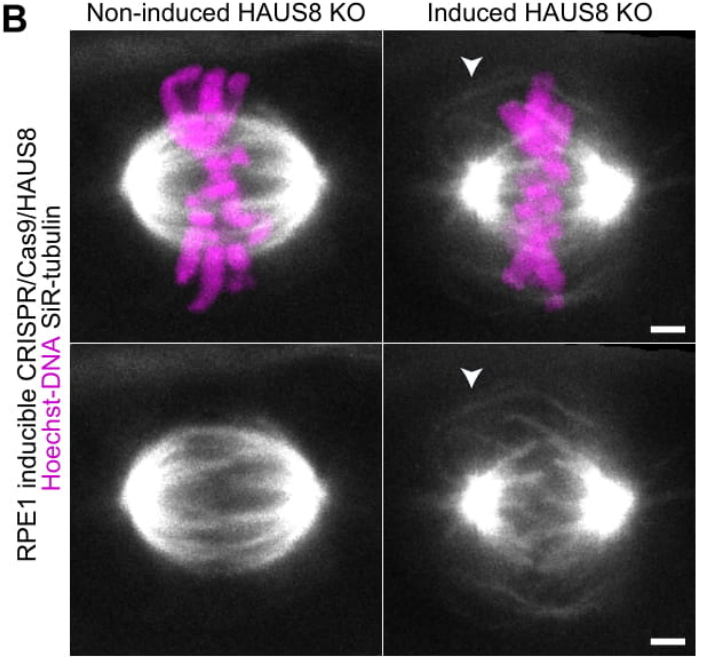

C

\begin{tabular}{|c|c|}
\hline Control & HAUS8 siRNA or CRISPR knock-out \\
\hline - Outermost bundle & - Bundle with bridging fiber — Bundle w/o bridging fiber - Bundle w/o kinetochores \\
\hline
\end{tabular}
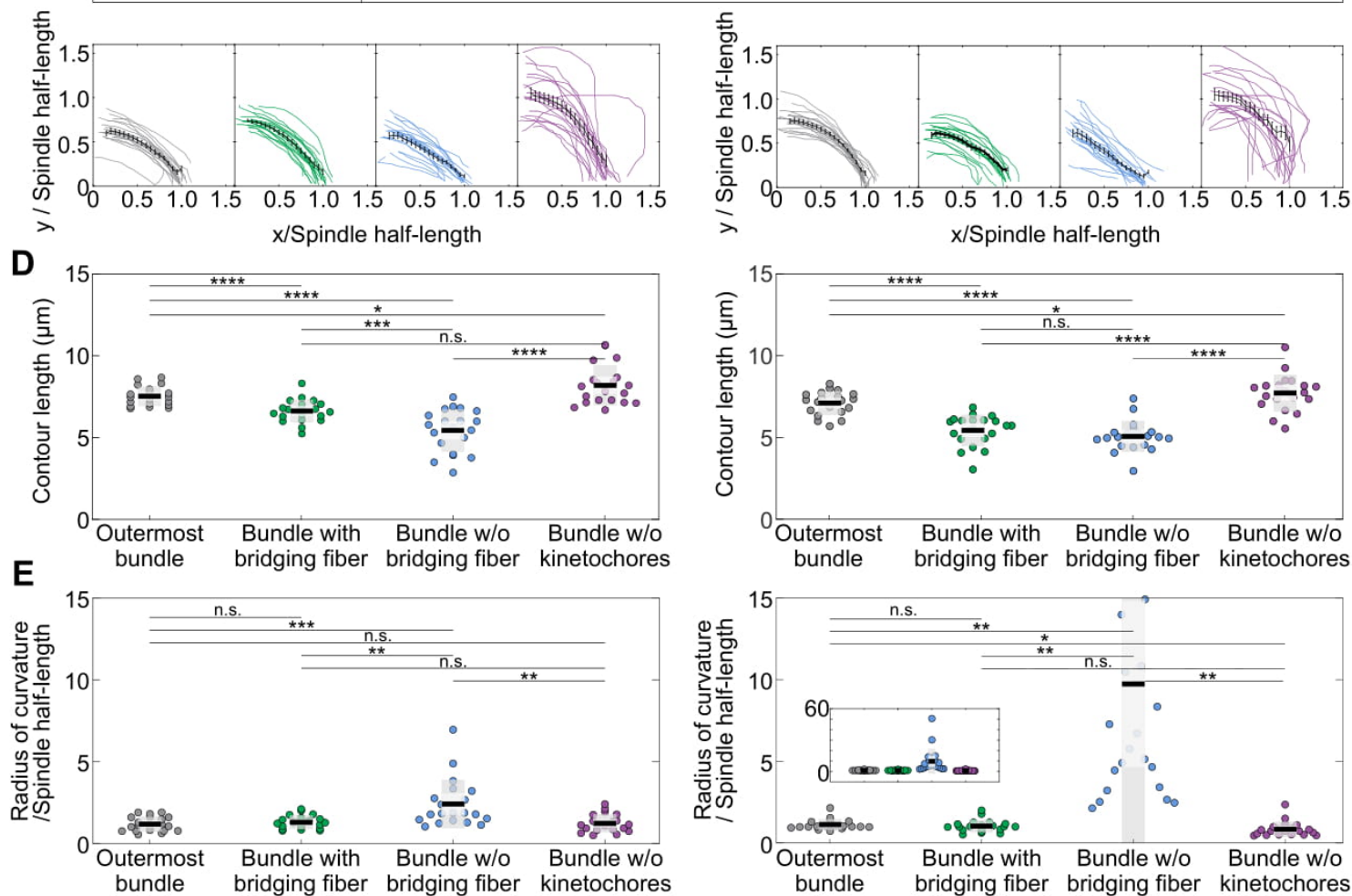

Figure 5. Bundle shapes change upon augmin depletion. (A) Live images (maximum projections of 3 central z-planes) of metaphase spindles in RPE1 cells stably expressing CENP-A-GFP and Centrin1-GFP (both in magenta) and stained with SiR-tubulin (gray). A long curved bundle without kinetochores in the HAUS8 siRNAtreated cell is marked with white arrowheads. (B) Live images (maximum projections of 3 central z-planes) of metaphase spindles in RPE1 inducible CRISPR/Cas9/HAUS8 KO cells stained with SiR-tubulin (gray) and Hoechst dye (magenta) for chromosomes. A long curved bundle without kinetochores is marked with white arrowheads. Scale bars in (A) and (B), $2 \mu \mathrm{m}$. (C) Bundle contours in cells from (A) and (B); mean \pm SEM is shown in black. (D) Univariate scatter plot of contour lengths and (E) radii of curvature (inset shows the complete data set). (C-E) Data on the left correspond to RPE1 cells stably expressing CENP-A-GFP and 
Centrin1-GFP and data on the right to RPE1 inducible CRISPR/Cas9/HAUS8 KO cells. Boxes represent standard deviation (dark gray), 95\% confidence interval of the mean (light gray) and mean value (black). All results were obtained from three independent experiments. Statistical analysis (D and E); t-test, p-value legend: < $0.0001(* * * *), 0.0001$ to $0.001(* * *), 0.001$ to $0.01(* *), 0.01$ to $0.05(*), \geq 0.05$ (ns).

Table 1. Number and shapes of microtubule bundles in control cells, HAUS6 and HAUS8 siRNA treated RPE1 cells stably expressing CENP-A-GFP and Centrin1-GFP, RPE1 inducible CRISPR/Cas9/HAUS8 knock-out (KO) cells and HeLa cells stably expressing PRC1-GFP and CENP-A-mCherry. All tracked bundles are outermost bundles. Values are given as mean $\pm \mathrm{SEM}$.

\begin{tabular}{|c|c|c|c|c|c|}
\hline $\begin{array}{l}\text { Cell } \\
\text { line }\end{array}$ & Treatment & Bundle type & $\begin{array}{c}\mathrm{N} \\
\text { (bundles) }\end{array}$ & $\begin{array}{l}\text { Contour } \\
\text { length } \\
(\mu \mathrm{m})\end{array}$ & $\begin{array}{c}\text { Radius of } \\
\text { curvature/spindle } \\
\text { half-length }\end{array}$ \\
\hline \multirow{11}{*}{ RPE1 } & control & $\begin{array}{c}\text { outermost } \\
\text { bundle }\end{array}$ & 20 & $7.5 \pm 0.1$ & $1.18 \pm 0.09$ \\
\hline & \multirow{3}{*}{$\begin{array}{l}\text { HAUS6 } \\
\text { siRNA }\end{array}$} & bridge & 10 & $6.8 \pm 0.1$ & $1.7 \pm 0.2$ \\
\hline & & no bridge & 10 & $6.4 \pm 0.2$ & $2.0 \pm 0.2$ \\
\hline & & no kinetochore & 10 & $8.2 \pm 0.8$ & $1.6 \pm 0.9$ \\
\hline & \multirow{3}{*}{$\begin{array}{c}\text { HAUS8 } \\
\text { siRNA }\end{array}$} & bridge & 20 & $6.6 \pm 0.5$ & $1.30 \pm 0.09$ \\
\hline & & no bridge & 20 & $5.4 \pm 0.3$ & $2.4 \pm 0.3$ \\
\hline & & no kinetochore & 20 & $8.2 \pm 0.3$ & $1.2 \pm 0.1$ \\
\hline & $\begin{array}{l}\text { non- } \\
\text { induced } \\
\text { KO }\end{array}$ & $\begin{array}{l}\text { outermost } \\
\text { bundle }\end{array}$ & 20 & $7.1 \pm 0.2$ & $1.13 \pm 0.07$ \\
\hline & \multirow{3}{*}{$\begin{array}{c}\text { HAUS8 } \\
\text { KO }\end{array}$} & bridge & 20 & $5.4 \pm 0.2$ & $1.04 \pm 0.08$ \\
\hline & & no bridge & 20 & $5.1 \pm 0.2$ & $10 \pm 3$ \\
\hline & & no kinetochore & 20 & $7.7 \pm 0.3$ & $0.9 \pm 0.1$ \\
\hline \multirow{4}{*}{$\mathrm{HeLa}$} & control & $\begin{array}{l}\text { outermost } \\
\text { bundle }\end{array}$ & 20 & $6.3 \pm 0.2$ & $0.90 \pm 0.05$ \\
\hline & \multirow{3}{*}{$\begin{array}{c}\text { HAUS6 } \\
\text { siRNA }\end{array}$} & bridge & 14 & $7.7 \pm 0.4$ & $1.27 \pm 0.09$ \\
\hline & & no bridge & 12 & $7.1 \pm 0.4$ & $7.1 \pm 1.8$ \\
\hline & & no kinetochore & 10 & $10.1 \pm 0.8$ & $1.0 \pm 0.2$ \\
\hline
\end{tabular}




\section{Augmin depletion slows down the poleward flux of microtubules}

Sliding of antiparallel microtubules in the central part of the spindle has been proposed to be involved in driving the poleward flux of microtubules (Brust-Mascher et al., 2009; Mitchison, 2005). Yet, the origin of poleward flux is still under debate, as other mechanisms including microtubule depolymerization at the pole and microtubule pushing against chromosome arms are also involved (Ganem et al., 2005; Steblyanko et al., 2020). Because of the significant perturbation of antiparallel bundles in augmin depleted cells, we set out to investigate the influence of augmin depletion on poleward flux (Fig. 6A). In HAUS6 siRNA treated RPE1 cells stably expressing $\alpha$-tubulin tagged with photoactivatable (PA) GFP, we observed a decreased photoactivation signal that was more evident in the inner part of the spindle close to the spindle axis (Fig. 6B, bottom time-lapse images, and Supplementary Video 5), corresponding to fewer microtubules present there. Interestingly, the poleward flux rate was reduced from $2.2 \pm 0.2 \mu \mathrm{m} / \mathrm{min}$ in control cells to $1.0 \pm 0.1 \mu \mathrm{m} / \mathrm{min}$ in HAUS6 siRNA treated cells (Fig. 6C and 6D). This result shows that the impairment of bridging fibers due to augmin depletion affects not only the spindle architecture, but also its dynamics, and suggests a role of augmin-nucleated bridging microtubules in the regulation of poleward flux. 
bioRxiv preprint doi: https://doi org/10.1101/2020.09.10.291740; this version posted November 19, 2020. The copyright holder for this preprint (which was not certified by peer review) is the author/funder, who has granted bioRxiv a license to display the preprint in perpetuity. It is made available under aCC-BY-NC-ND 4.0 International license.

A Expected outcomes after photoactivation (HAUS6 siRNA)

B

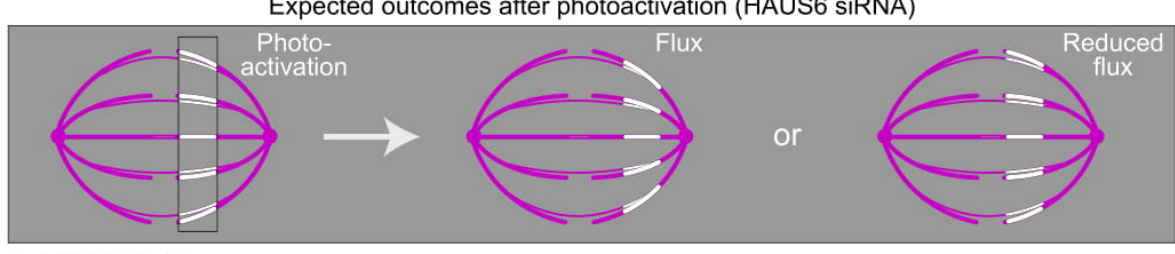

B RPE1 PA-tubulin SiR-tubulin
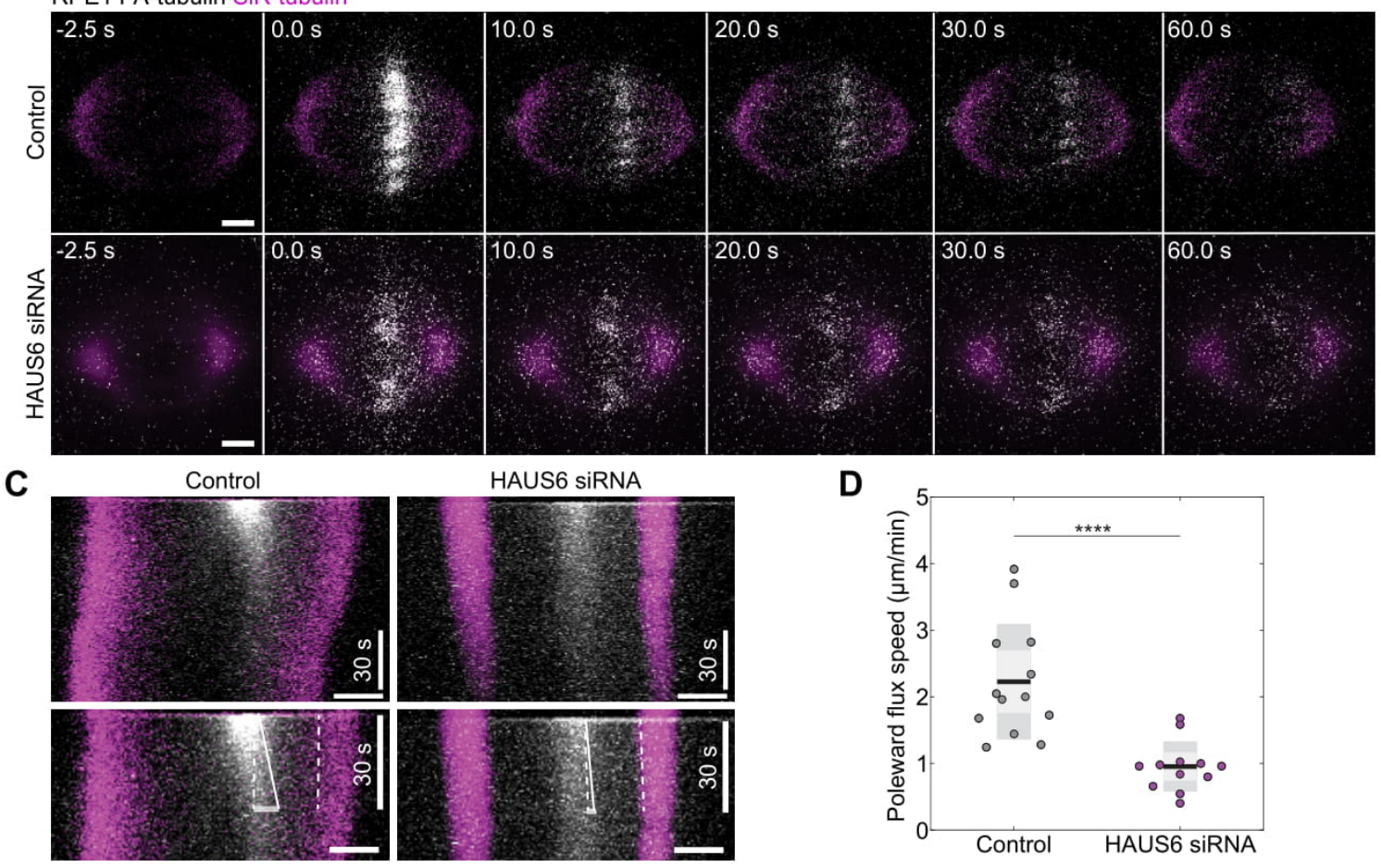

D

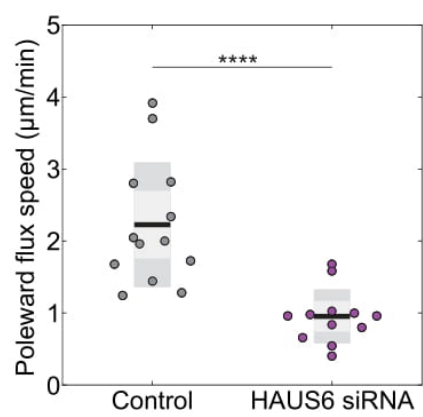

Figure 6. Augmin depletion decreases the poleward flux rate. (A) Schematic representation of expected outcomes after photoactivation (white segments) of spindles in cells depleted of HAUS6. (B) Time-lapse images of control (top) and HAUS6 (bottom) depleted RPE1 cells stably expressing PA-GFP- $\alpha$-tubulin and stained with SiR-tubulin (magenta) before $(-2.5 \mathrm{~s})$, at time 0 when photoactivation (white) was performed, and afterwards. (C) Kymograph of the spindles in (B) showing two spindle poles (magenta) and photoactivated regions (white). Poleward flux speed was measured during $30 \mathrm{~s}$ after photoactivation (bottom). The dashed line follows the spindle pole, the oblique solid line follows the outer border of the highest tubulin signal trail, and the horizontal line was used to obtain the poleward flux speed $(\Delta x / \Delta t)$. Horizontal scale bars in (B) and (C), $2 \mu \mathrm{m}$. (D) Univariate scatter plot of the poleward flux speed in control cells (gray) and after HAUS6 depletion (magenta). Boxes represent standard deviation (dark gray), $95 \%$ confidence interval of the mean (light gray) and mean value (black). All results were obtained from three independent experiments. Statistical analysis; t-test, p-value legend: $<0.0001(* * * *)$. 


\section{Discussion}

In this paper we provide evidence that the augmin complex is the major contributor to the nucleation and maintenance of bridging fibers. We propose a model of the metaphase spindle in which the bridging fiber, which laterally connects sister k-fibers, is formed by augminbased nucleation of microtubules along the existing kinetochore microtubules. The newly nucleated microtubules create an antiparallel overlap with the microtubules extending from the opposite side of the spindle. Within this overlap, microtubules slide apart, generating a pushing force that the bridging fiber exerts on its k-fibers, thereby producing tension between sister kinetochores and poleward flux of the k-fibers (Fig. 7).

Involvement of the augmin complex in generation of spindle microtubules has been extensively studied (Song et al., 2018; Uehara et al., 2009), and its depletion has been implicated in several defects of spindle architecture and function, including reduced spindle stability, smaller interkinetochore distance, chromosome misalignment and the formation of long, curved bundles (Hayward et al., 2014; Uehara and Goshima, 2010; Uehara et al., 2016; Uehara et al., 2009; Wu et al., 2008; Zhu et al., 2008). Our work shows that the depletion of the augmin complex by silencing the HAUS6 or HAUS8 subunits causes severe thinning of bridging fibers in metaphase spindles, especially in the inner part of the spindle close to the pole-to-pole axis. K-fibers were also thinner, though to a lesser extent. We conclude that the bridging microtubules are predominantly nucleated by the augmin complex along the preexisting microtubules, whereas k-fiber microtubules are mainly nucleated in an augminindependent manner, most likely by the centrosome. This is in agreement with previous electron microscopy studies of mammalian spindles, where k-fiber microtubules were observed to extend from the centrosome (McDonald et al., 1992), whereas interpolar microtubules, which likely correspond to bridging fibers, start at different points along the kfiber (Mastronarde et al., 1993). Moreover, recent electron tomography of spindles in RPE1 cells confirmed this result by showing that microtubule minus ends are found along the kfiber, less than $50 \mathrm{~nm}$ from the k-fiber wall and at a distance $2-4 \mu \mathrm{m}$ from the pole (O'Toole et al., 2020). We propose that the existing kinetochore microtubules orient the growth of augmin-nucleated microtubules, which later become kinetochore microtubules if their plus end binds to the kinetochore or bridging microtubules if they grow past the kinetochore and interact with the bridging or kinetochore microtubules on the other side.

Previous work showed that augmin depletion results in a decrease of interkinetochore distance (Uehara et al., 2009; Zhu et al., 2008), a readout of tension, but it remained unclear if this is 
due to impaired k-fibers or perturbation of other microtubules. Treatment of cells with a microtubule-destabilizing agent that results in thinner k-fibers causes a reduction of the interkinetochore tension (Dudka et al., 2018), supporting the former possibility. However, a similar effect on interkinetochore tension was observed upon perturbation of the bridging fiber by removing the microtubule crosslinker PRC1 (Jagrić et al., 2020; Kajtez et al., 2016; Polak et al., 2017), in agreement with the latter possibility. Our observation that the interkinetochore tension was most severely affected in the inner part of the spindle, where the bridging fibers were most impaired, suggests a link between interkinetochore tension and augmin-generated bridging microtubules. Furthermore, when looking at a subset of kinetochore pairs that had a bridging fiber and those that did not, we found that the tension was more compromised in the latter group. Notably, tension was independent of the k-fiber thickness within each group. Although our experiments cannot discern the relative contribution of the bridging and k-fiber impairment in the decrease of tension on kinetochores, they reveal that augmin-generated bridging microtubules have a significant and $\mathrm{k}$-fiber independent role in the maintenance of interkinetochore tension.

One of the most prominent effects we observed in augmin depleted cells, in addition to the thinning of bridging fibers, was a particular spatial organization of the remaining bridging fibers, which localized at the spindle edge in a barrel-like arrangement. Nevertheless, DNA and kinetochores were evenly distributed throughout the spindle cross-section in a manner similar to control cells, suggesting that bridging fibers do not strongly influence this distribution of kinetochores over the spindle cross-section. The majority of kinetochore pairs with reduced bridging fibers were found in the inner part of the spindle close to the pole-topole axis, which is in agreement with the spatial distribution of PRC1-labeled bridging fibers preferentially at the spindle periphery. The overlap regions of these bridging fibers were longer than in control cells, possibly due to the activity of residual PRC1 crosslinking the remaining microtubules formed by alternative nucleation pathways. Similarly, the extra-long curved interpolar bundles without kinetochores, which are found at the periphery of the spindle, likely arise due to residual PRC1 together with the excess of free tubulin present as a consequence of less tubulin being incorporated in the spindle.

The overall shape of the spindle became more diamond-like after augmin depletion because the outermost k-fibers were straight rather than rounded. This straight shape is indicative of decreased compression within the k-fibers and demonstrates a role of augmin-generated 
bridging microtubules in the regulation of forces not only on kinetochores but also within microtubule bundles, and consequently in the maintenance of the spindle shape.

Interestingly, augmin depletion results in slower microtubule poleward flux, which supports the model in which poleward flux is mainly driven by sliding apart of antiparallel microtubules (Brust-Mascher et al., 2009; Mitchison, 2005; Miyamoto et al., 2004). Microtubule sliding in metaphase may be similar to the situation in early anaphase, where the sliding forces within the bridging fiber push the attached k-fibers apart, driving separation of sister kinetochores and spindle elongation (Vukušić et al., 2017). In agreement with this strong interaction between bridging and k-fibers in anaphase, experiments on metaphase spindles based on laser ablation, microneedle pulling, and depletion of crosslinking proteins PRC1 and NuMa showed that the bridging fiber and the corresponding k-fibers are tightly linked into a single mechanical element (Elting et al., 2017; Kajtez et al., 2016; Suresh et al., 2020). We propose that the sliding forces within the bridging fiber are transmitted onto the attached sister k-fibers through this crosslinking. The resulting pushing apart of sister k-fibers contributes to the poleward flux of k-fibers and the interkinetochore tension (Fig. 7).

Considering the importance of the interkinetochore tension for the accuracy of cell division (Lampson and Grishchuk, 2017), the maintenance of tension by the bridging fiber might also represent an important mechanism for silencing of the spindle assembly checkpoint (Musacchio and Salmon, 2007; Nicklas et al., 1995; Taylor et al., 1998). As augmin depletion causes a higher incidence of aneuploidy (Wu et al., 2008), it is conceivable that weakening of bridging fibers contributes to this issue.

Our results from metaphase open an interesting question about how, during spindle assembly in prometaphase, the bridging fiber and the k-fiber form and start to interact. The attachment of kinetochores to k-fibers and their biorientation may precede the formation of the bridging fiber, but the alternative scenario in which the kinetochores interact laterally with the bridging fiber before both k-fibers form and the kinetochores become bioriented is also possible (Simunić and Tolić, 2016). Our finding that the bridging microtubules in metaphase are predominantly nucleated by augmin indicates a need for preexisting microtubules for their nucleation, which may be k-fibers or a subset of bridging microtubules formed at the centrosome. It will be interesting to explore to what extent the cell uses these scenarios of microtubule bundle formation to build the spindle. 

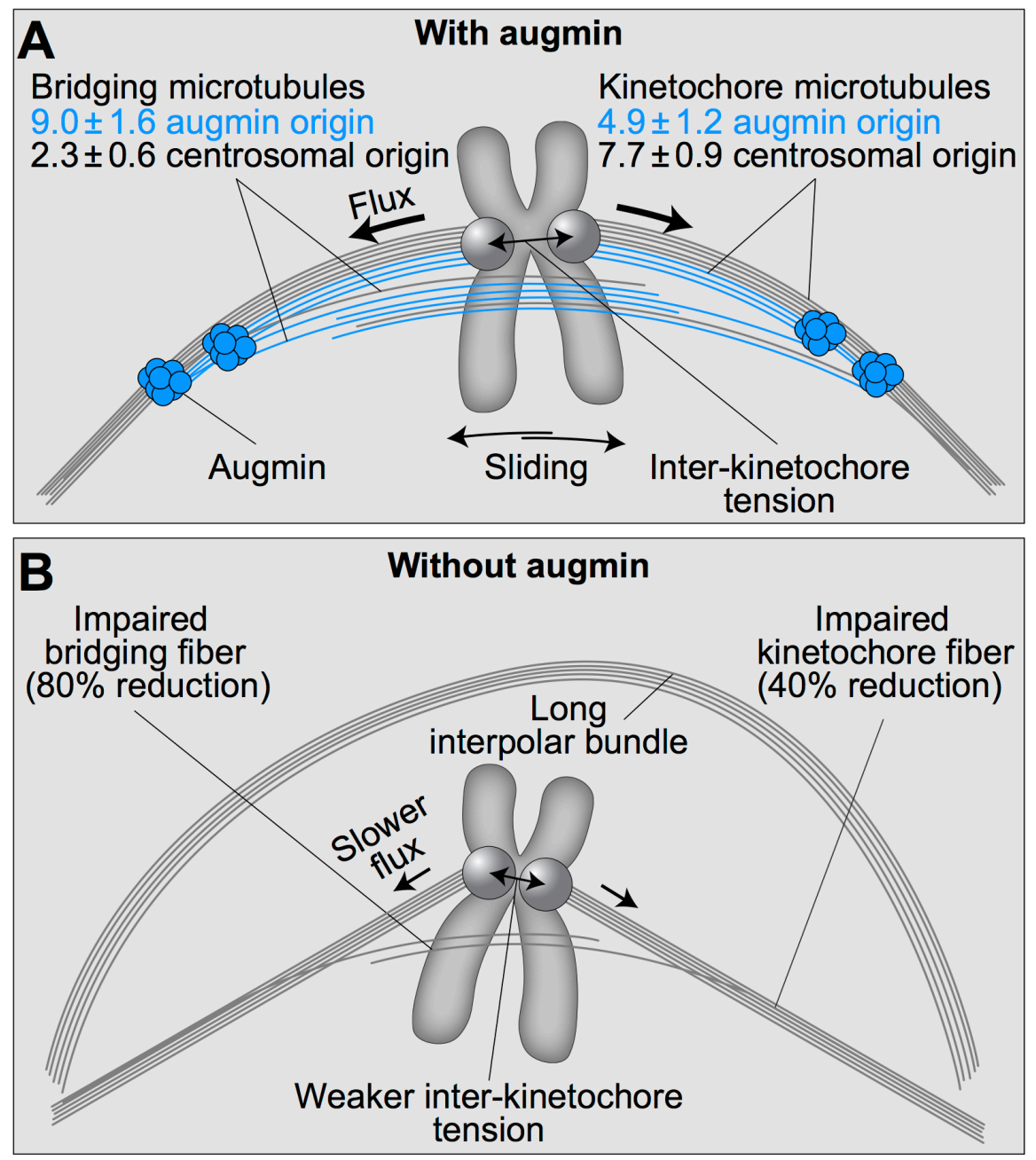

Figure 7. A model of augmin-dependent nucleation of bridging microtubules and their contribution to interkinetochore tension and poleward flux. (A) Bridging microtubules are mainly formed by augmindependent nucleation. Within their overlaps microtubules slide apart, creating a pushing force onto the k-fibers, thereby producing tension between sister kinetochores and poleward flux of k-fibers. Augmin-nucleated microtubules (blue) and centrosome-nucleated microtubules (gray) in bridging and k-fibers are shown together with the number of microtubules in each group estimated from HAUS6 depletion experiments. (B) Augmin depletion leads to a strong reduction of the microtubule number in the bridging fiber and to a significant but smaller reduction in the k-fibers, slower poleward flux, and weaker interkinetochore tension, with the characteristic long interpolar bundles appearing on the spindle periphery. 


\section{MATERIALS AND METHODS}

Cell lines. Experiments were carried out using unlabeled human HeLa-TDS cells from the High-Throughput Technology Development Studio (MPI-CBG, Dresden); human HeLaKyoto BAC cells stably expressing PRC1-GFP, courtesy of Ina Poser and Tony Hyman (MPICBG, Dresden, Germany); human HeLa-Kyoto BAC cells stably expressing PRC1-GFP and CENP-A-mCherry, derived from the previously described HeLa-PRC1-GFP cell line using a lentiviral vector system; human HeLa cells stably expressing CENP-A-GFP and Centrin1GFP, which were a gift from Emanuele Roscioli and Andrew McAinsh (University of Warwick, Coventry, UK); human HeLa-TDS cells stably expressing GFP- $\alpha$-tubulin as described in our previous work (Kajtez et al., 2016); human hTERT-RPE1 (hTERT immortalized retinal pigment epithelium) cells stably expressing CENP-A-GFP and Centrin1GFP, a courtesy of Alexey Khodjakov (Wadsworth Center, New York State Department of Health, Albany, NY, USA; human hTERT-RPE1 inducible CRISPR/Cas9/HAUS8 knock-out (KO) cells, which were a gift from Iain Cheeseman (Massachusetts Institute of Technology, Cambridge, MA, USA) and human hTERT-RPE1 cells stably expressing PA-GFP- $\alpha$-tubulin, a gift from Patrick Meraldi (Faculty of Medicine, University of Geneva, Switzerland).

Cell culture. Unlabeled HeLa-TDS cells, HeLa-Kyoto BAC cells stably expressing PRC1GFP, HeLa-Kyoto BAC cells stably expressing PRC1-GFP and CENP-A-mCherry, human RPE1 cells stably expressing CENP-A-GFP and Centrin1-GFP, RPE1 inducible CRISPR/Cas9/HAUS8 KO cells and human RPE1 cells stably expressing PA-GFP- $\alpha$-tubulin were cultured in flasks in Dulbecco's Modified Eagle's Medium with $1 \mathrm{~g} / \mathrm{L}$ D-glucose, pyruvate and L-glutamine (DMEM, Lonza, Basel, Switzerland), supplemented with 10\% (vol/vol) heat-inactivated Fetal Bovine Serum (FBS, Sigma Aldrich, St. Louis, MO, USA) and penicillin $(100 \mathrm{IU} / \mathrm{mL}) /$ streptomycin $(100 \mathrm{mg} / \mathrm{mL})$ solution (Lonza, Basel, Switzerland). For the selection of HeLa PRC1-GFP, Hela PRC1-GFP CENP-A-mCherry, HeLa CENP-AGFP Centrin1-GFP and Hela GFP- $\alpha$-tubulin cell lines, $50 \mu \mathrm{g} / \mathrm{ml}$ geneticin was added to the medium (Life Technologies, Waltham, MA, USA), whereas $500 \mu \mathrm{g} / \mathrm{ml} \mathrm{G418} \mathrm{was} \mathrm{added} \mathrm{to}$ the medium for the selection of RPE1 PA-GFP- $\alpha$-tubulin cell line.

The induction of human RPE1 inducible CRISPR/Cas9/HAUS8 KO cell line was performed every 24 hours for four days using $1 \mu \mathrm{g} / \mathrm{ml}$ doxycycline hyclate (Sigma Aldrich, St. Louis, $\mathrm{MO}, \mathrm{USA}$ ), with cells being imaged on the fifth day. All cells were kept at $37^{\circ} \mathrm{C}$ and $5 \% \mathrm{CO}_{2}$ in a Galaxy 170 R humidified incubator (Eppendorf, Hamburg, Germany). All cell lines have been regularly tested for mycoplasma contamination by examining the samples for 
extracellular DNA staining with SiR-DNA (100 nM, Spirochrome, Stein am Rhein, Switzerland) and Hoechst 33342 dye (1 drop/2 ml of NucBlue Live ReadyProbes Reagent, Thermo Fisher Scientific, Waltham, MA, USA) and have been confirmed to be mycoplasma free.

Lentiviral cell transfection. For stable expression of CENP-A in HeLa PRC1-GFP cells, a lentiviral transduction was used. Lentivirus was produced in HEK-293 cells by cotransfection of the cells with pLenti6-CENP-A-mCherry (No. 89767, Addgene) plasmid carrying blasticidin selection, pSPAX2 (No. 12260, Addgene) and pCMV-VSV-G (No. 8454, Addgene) plasmids. After 36 and 72 hours, the culture medium containing lentivirus particles was harvested, filtered through a $20 \mu \mathrm{m}$ pore size filter and added to HeLa cells stably expressing PRC1-GFP. After the second treatment, HeLa cells were cultured for six weeks under blasticidin selection prior to being used for imaging.

Sample preparation and RNAi transfection. At $80 \%$ confluence, DMEM medium was removed from the flask and cells were washed with $5 \mathrm{ml}$ of PBS. Then, $1 \mathrm{ml} 1 \%$ trypsin/EDTA (Biochrom AG, Berlin, Germany) was added to the flask and cells were incubated at $37^{\circ} \mathrm{C}$ and $5 \% \mathrm{CO}_{2}$ in a humidified incubator for 5 minutes. After the incubation, trypsin was blocked by adding $2 \mathrm{ml}$ of DMEM medium. For RNAi experiments, the cells were seeded to reach $60 \%$ confluence the next day and cultured on $35 \mathrm{~mm}$ uncoated dishes with 0.16-0.19 mm (1.5 coverglass) glass thickness (MatTek Corporation, Ashland, MA, USA) in $2 \mathrm{~mL}$ DMEM medium with previously described supplements. After one day of growth, cells were transfected with either targeting or non-targeting siRNA constructs which were diluted in OPTI-MEM medium (Life Technologies, Waltham, MA, US) to a final concentration of $20 \mathrm{nM}$ in medium with cells. All transfections were performed 48 hours prior to imaging using Lipofectamine RNAiMAX Reagent (Life Technologies, Waltham, MA, US) according to the instructions provided by the manufacturer. After four hours of treatment, the medium was changed to the previously described DMEM medium. The constructs used were human HAUS6 siRNA (L-018372-01-0005, Dharmacon, Lafayette, CO, USA), human HAUS8 siRNA (L-031247-01-0005, Dharmacon, Lafayette, CO, USA) and control siRNA (D-001810-10-05, Dharmacon, Lafayette, CO, USA).

In order to visualize microtubules, all cells except HeLa PRC1-GFP and HeLa GFP- $\alpha$-tubulin cells were stained to a final concentration of $100 \mathrm{nM}$ with a far-red silicon rhodamine (SiR)tubulin-670 dye (Spirochrome, Stein am Rhein, Switzerland), 45 minutes to 2 hours prior to imaging. In order to avoid dye efflux, a broad-spectrum efflux pump inhibitor verapamil 
(Spirochrome, Stein am Rhein, Switzerland) was added at a final concentration of $0.5 \mu \mathrm{M}$ to RPE1 cells along with SiR-tubulin. For chromosome visualization, HeLa PRC1-GFP and HeLa GFP- $\alpha$-tubulin cells were stained with 100 nM SiR-DNA dye for 20 minutes to 2 hours prior to imaging. RPE1 PA-GFP- $\alpha$-tubulin cells were stained with $25 \mathrm{nM}$ SiR-tubulin for 3 hours prior to imaging and RPE1 inducible CRISPR/Cas9/HAUS8 KO cells were stained with 1 drop of Hoechst 33342 dye (Thermo Fisher Scientific, Waltham, MA, USA) in $2 \mathrm{~mL}$ of DMEM medium.

Immunocytochemistry. HeLa cells stably expressing PRC1-GFP and RPE1 inducible CRISPR/Cas9/HAUS8 KO cells were grown on glass-bottom dishes (14 mm, No. 1.5, MatTek Corporation) and fixed by $2 \mathrm{ml}$ of ice-cold methanol for $1 \mathrm{~min}$ at $-20^{\circ} \mathrm{C}$. Following fixation, cells were washed 3 times for 5 minutes with $1 \mathrm{ml}$ of PBS and permeabilized with $0.5 \%$ Triton-X-100 in water for 15 minutes at a room temperature. This step was repeated twice when tubulin staining was preformed. To block unspecific binding, cells were incubated in $1 \mathrm{ml}$ of blocking buffer ( $1 \%$ normal goat serum (NGS)) for 1 hour at $4{ }^{\circ} \mathrm{C}$. Cells were then washed 3 times for 5 minutes with $1 \mathrm{ml}$ of PBS and incubated with $250 \mu \mathrm{l}$ of primary antibody solution overnight at $4{ }^{\circ} \mathrm{C}$. The primary antibodies used were as follows: rabbit polyclonal PRC1 (diluted 1:100, sc-8356, Santa Cruz Biotechnology), rabbit polyclonal HAUS6 (diluted 1:250, ab-150806, Abcam), rabbit polyclonal HAUS8 (diluted 1:100, PA521331, Invitrogen) and rat monoclonal tubulin (diluted 1:100, MA1-80017, Invitrogen). After the incubation with a primary antibody, cells were washed 3 times for 5 minutes with $1 \mathrm{ml}$ of PBS and then incubated with $250 \mu \mathrm{l}$ of secondary antibody for 45 minutes at a room temperature. Alexa Fluor 488 and 594 (Abcam) were used as secondary antibodies at a 1:1000 dilution for PRC1 staining, 1:500 dilution for HAUS6 and 1:250 for HAUS8 and tubulin staining. DAPI $(1 \mu \mathrm{g} / \mathrm{mL})$ was used for chromosome visualization.

Immunoblotting. RPE1 cells stably expressing CENP-A-GFP and Centrin1-GFP and HeLa cells stably expressing PRC1-GFP were grown on six-well plates (Greiner Bio-one) and subjected to HAUS6 or HAUS8 siRNA treatment as indicated before. Following transfection, cells were lysed in RIPA buffer (R0287, Sigma) containing 1x protease inhibitor (5892970001, Roche), 1x phosphatase inhibitor (4906837001, Roche) and 1mM PMSF by two cycles of freezing and thawing in liquid nitrogen. Protein extracts were mixed with $2 \mathrm{x}$ Laemlli sample buffer (S3401, Sigma) and heated at $95^{\circ} \mathrm{C}$ for 10 minutes prior to SDS-PAGE. After protein transfer onto the nitrocellulose membrane (IB23002, Invitrogen) and blocking with blocking solution (5\% bovine serum albumin and 0,1\% Tween 20 in PBS) for 1 hour, 
membranes were incubated overnight at $4^{\circ} \mathrm{C}$ with primary antibodies diluted in blocking solution. The primary antibodies used were as follows: rabbit polyclonal HAUS6 (diluted 1:250, ab-150806, Abcam), rabbit polyclonal GAPDH (diluted 1:100, ab9485, Abcam). Rabbit polyclonal HAUS8 antibody (diluted 1:100, PA5-21331, Invitrogen) resulted in no detectable bands under these conditions. Membranes were washed with $0.1 \%$ Tween 20 in PBS, incubated for 1 hour with anti-rabbit HRP-conjugated secondary antibodies (dilution 1:15000, ab6721) and visualized on the C-DiGit blot scanner (LI-COR, Bad Homburg, Germany) with WesternSure PREMIUM Chemiluminescent Substrate (926-95000, LI-COR).

Microscopy. Cells were imaged using a previously described microscope setup (Buda et al., 2017), consisting of a Bruker Opterra Multipoint Scanning Confocal Microscope (Bruker Nano Surfaces, Middleton, WI, USA), mounted on a Nikon Ti-E inverted microscope with a Nikon CFI Plan Apo VC 100x/1.4 numerical aperture oil objective (Nikon, Tokyo, Japan). During live-cell imaging, cells were kept at $37^{\circ} \mathrm{C}$ and $5 \% \mathrm{CO}_{2}$ in Okolab Cage Incubator (Okolab, Pozzuoli, NA, Italy). To excite Hoechst, GFP, mCherry or SiR fluorescence, a 405 $\mathrm{nm}, 488 \mathrm{~nm}, 561 \mathrm{~nm}$ or $647 \mathrm{~nm}$ laser lines were used, respectively. Opterra Dichroic and Barrier Filter Set 405/488/561/640 enabled the separation of excitation light from the emitted fluorescence. Images were acquired using Evolve 512 Delta Electron Multiplying Charge Coupled Device (EMCCD) Camera (Photometrics, Tuscon, AZ, USA), with camera readout mode of $20 \mathrm{MHz}$. The xy pixel size was $83 \mathrm{~nm}$. In all experiments where the whole spindle stack was imaged, z-stacks were acquired with unidirectional xyz scan mode at 37 focal planes and $0.5 \mu \mathrm{m}$ distance between planes. For time lapse imaging, a single $\mathrm{z}$ plane was acquired every 0.5 seconds. Photoactivation was performed perpendicular to pole-to-pole axis of metaphase spindles using a 405-nm laser diode (Coherent, Santa Clara, CA, USA) and a line pattern of 12 equally distributed points, with each point representing one laser hit. The interval between the points was $0.05 \mathrm{~ms}$ and photoactivation area was set to $0.5 \mu \mathrm{m}$ for each point. The interval between successive frames was set to $0.5 \mathrm{~s}$, one central $\mathrm{z}$-plane was imaged and 160 successive frames were recorded, thereby obtaining an $80 \mathrm{~s}$ time lapse for each cell.

Image processing and data analysis. In cells where HAUS6 or HAUS8 were silenced only bipolar metaphase spindles were imaged and analyzed, even though multipolar spindles were observed as reported previously (Lawo et al., 2009). For experiments that included a transformation of spindles into the end-on view, only cells that had spindles with both poles approximately in the same plane were imaged and analyzed. All images were analyzed in 
Fiji/ImageJ (National Institutes of Health, Bethesda, MD, USA). Raw images were used for quantification and contrast was adjusted for clarity for representation on figures. MatLab (MathWorks, Natick, MA, USA) was used to create the plots, Rstudio (R Foundation for Statistical Computing, Vienna, Austria) to transform the cells into an end-on view and Python to fit the circles to determine the curvature of bundles. Figures were assembled in Adobe Illustrator CS5 (Adobe Systems, Mountain View, CA, USA).

Measuring the bridging fiber intensity. Tubulin intensity of a bridging fiber and k-fiber region was measured in a single $\mathrm{z}$ plane using the Segmented Line tool by drawing a 5 pixel line along the contour of k-fibers and the corresponding bridging fiber. Background was measured in the same $\mathrm{z}$ plane by drawing the 5 pixel line along the length of the metaphase midzone, as this represents the background signal caused by the presence of neighboring microtubules. The minimum value of the midzone intensity profile was subtracted from the intensity profiles of bundle contours. The minimum value of the mean intensity profile was set as a distance of zero $\mu \mathrm{m}$ and was selected as the center of the bridging fiber. The final intensity of a bridging fiber $\left(I_{\mathrm{b}}\right)$ was calculated as the mean value of intensities in the area 500 $\mathrm{nm}$ around the center of the bridging fiber. The final intensity of a k-fiber region $\left(I_{\mathrm{bk}}\right)$, which also includes the bridging fiber, was calculated as an average of two mean values of intensities in the area $500 \mathrm{~nm}$ around the distance of $1.5 \mu \mathrm{m}$ away from the center of the bridging fiber. The intensity value of k-fibers alone $\left(I_{\mathrm{k}}\right)$ was then calculated as $I_{\mathrm{k}}=I_{\mathrm{bk}}-I_{\mathrm{b}}$.

For the analysis based on small square regions, tubulin intensity in bridging and k-fibers was measured using a 5x5 pixel Square tool. To measure bridging fibers, the square was positioned on the fiber spanning the area between two kinetochores. K-fibers were measured right next to the kinetochores. The average intensity of the two sister k-fibers was used for further analyses. The background was measured using the same tool right above and below the bridging fiber and the average intensity of the two regions was subtracted from bridging and k-fiber intensities.

Comparison of SiR- and GFP-tubulin signal. For the comparison of SiR and GFP-tubulin signal in HeLa cells stably expressing GFP- $\alpha$-tubulin, the previously described analysis was used. Tubulin intensity of a bridging fiber and k-fiber region was measured in a single $\mathrm{z}$ plane using the Segmented Line tool by drawing the 5 pixel line along the contour of k-fibers and the corresponding bridging fiber. Due to high noise, only outermost bundles were taken into analysis and the reduction was not calculated. The background was measured in the same $\mathrm{z}$ plane by drawing the $5 \times 5$ pixel square in the cytoplasm and was subtracted from the bundle 
intensity profiles. The minimum value of the intensity profile was set as a distance of zero $\mu \mathrm{m}$ and was selected as the center of the bridging fiber.

The interkinetochore distance. The interkinetochore distance was measured in ImageJ using a Point tool, after the images were rotated to make the spindle parallel to x-axis. Two points were put on spindle poles and two points on the upper and lower edge of the metaphase plate. Additional two points were put on the centers of signal in each kinetochore pair. Interkinetochore distance and distance from spindle axes were calculated using a homewritten MatLab script. Two-dimensional analysis was applied when all tracked kinetochore pairs resided within $2 \mu \mathrm{m}$ around the central z-plane, which was determined as the plane in which both centrosomes were clearly visible. Three-dimensional analysis was used when all kinetochore pairs in the spindle were taken into account, regardless of their position. In 2D analysis, Line tool was used to measure the projected distance of a middle point between two sister kinetochores in a particular z-plane from the long spindle axis. In 3D analysis, the exact distance from the long spindle axis (c) was calculated using the Pythagorean theorem, where a $=$ projected distance of a middle point between two sister kinetochores in a particular z-plane from the long spindle axis, $b=$ distance between the central z-plane and the z-plane of the kinetochore pair x 0.81 (correction factor, see Transformation of spindles into an end-on view).

Additionally, kinetochores with visible bridging fibers and kinetochores with no visible bridging fibers were separately tracked and analyzed in RPE1 cells stably expressing CENPA-GFP and Centrin1-GFP. The presence of bridging fibers was determined by measuring intensity profiles of the tubulin signal between two kinetochores.

Transformation of spindles into an end-on view. Z-stacks of the mitotic spindles that were positioned horizontally were transformed into the end-on view using a home-written $\mathrm{R}$ script previously described in our work (Novak et al., 2018). Prior to transformation, a singlechannel grayscale z-stack was rotated using ImageJ to make the long axis of the spindle parallel to the x-axis. Signal intensity at each pixel was used to obtain an end-on view of the spindles by applying the following transformation: I' $(\mathrm{i} \cdot$ pixel size, $\mathrm{j} \cdot$ pixel size, $\mathrm{k} \cdot \mathrm{z}-$ distance $)=I(\mathrm{k} \cdot \mathrm{z}$-distance, $\mathrm{i} \cdot$ pixel size, $\mathrm{j} \cdot$ pixel size $)$. A correction factor of 0.81 was used for the z-distance to correct for aberrations caused by the different refractive index mismatch of aqueous samples and immersion oil (Novak et al., 2018). 
Number of bundles. The number of bundles in HeLa-PRC1-GFP cells was determined in an end-on view of the spindle by using sum intensity projections of 10 central z-planes covering $0.83 \mu \mathrm{m}$ along the long spindle axis. Intensity profiles of PRC1-GFP were measured on the same sum intensity projections by drawing a 50 pixel wide Straight line tool across the diameter of the spindle.

Spindle length, width and metaphase plate diameter. Spindle length, width and metaphase plate diameter were measured on maximum intensity projections of the side view z-stack. Spindle length was determined in ImageJ by drawing the line from pole to pole using the Straight line tool. The position of the poles was determined as either the center of Centrin1 signal or the outermost points of the tubulin or PRC1 signal at the spindle pole. Spindle width was measured as the distance between two lines parallel to the long axis of the spindle and encompassing the outermost PRC1- or tubulin-labeled bundles. Additionally, in RPE1 cells stably expressing CENP-A-GFP and Centrin1-GFP the metaphase plate diameter was measured as the distance between the outermost kinetochore pairs, whereas in HeLa PRC1GFP it was measured as the distance between the outermost chromosome ends after specifying the metaphase plate using Square Tool.

Overlap length. Overlap length was measured on sum intensity projections of 2 to 4 z-planes covering the entire bundle of interest, using ImageJ Segmented line tool by drawing a pole-topole line along the contour of PRC1-GFP and acquiring an intensity profile. The overlap length was defined as the length of the base of the PRC1-GFP intensity peak (Polak et al., 2017).

PRC1 intensity. PRC1 intensity was measured in sum intensity projections of all z-planes covering the whole spindle. Total PRC1 signal in the cell was marked by using Polygon selection tool and $5 \times 5$ Square tool was used to determine the background in the cytoplasm. The final intensity values were obtained using the following formula: PRC1 intensity = Integrated Density of the spindle - (Area of selected cell x mean fluorescence of background). Additionally, an intensity profile was acquired by drawing a $2.5 \mu \mathrm{m}$ wide Line toll across the spindle cross-section in an end-on view.

Spindle shape. The shape of spindles was determined in ImageJ using a Point tool. The images were rotated to make spindle long axis parallel to the $\mathrm{x}$-axis. A line that goes from the top to the bottom of a metaphase plate and a pole-to-pole line were drawn using a Line tool. The meeting point of the lines was defined as a middle point of the spindle. Then, one point 
was put in the middle of the spindle, two points on spindle poles and two points on the upper and lower edge of the metaphase plate. Additional points were used to track the outermost bundle contours at least $2.5 \mu \mathrm{m}$ away from the long spindle axis in single z-slices. The first point of the bundle was at a spindle pole and the last point was either next to the kinetochore or at half of the long spindle axis. The outermost bundle was tracked in control cells. In treated cells, three different groups of outermost bundles were tracked: bundles with visible bridging fibers, bundles with no visible bridging fibers and curved bundles extending far from the metaphase plate. Shape and curvature were calculated using a home-written Python script based on a previously published method (Kanatani and Rangarajan, 2011). Contour lengths of the bundles were measured by calculating the distance between the first and the last point of the tracked bundle.

Poleward flux rate. To measure the poleward flux, a line covering the whole spindle width was drawn from pole to pole using the Line tool. Kymograph Builder was used to obtain kymographs and poleward flux was measured as previously described (Pereira and Maiato, 2010) during the first 30 seconds after photoactivation along the outer border of the highest intensity signal trail and towards the closest spindle pole.

\section{Acknowledgements}

We thank Alexey Khodjakov for the hTERT-RPE1 CENP-A-GFP Centrin1-GFP cell line, Ina Poser and Tony Hyman for the HeLa PRC1-GFP cell line, Emanuele Roscioli and Andrew McAinsh for the HeLa CENP-A-GFP Centrin1-GFP cell line, Mariola Chacon for the HeLaTDS cell line, Iain Cheeseman for the hTERT-RPE1 inducible CRISPR/Cas9/HAUS8 KO cell line and Patrick Meraldi for the hTERT-RPE1 PA-GFP- $\alpha$-tubulin cell line, Arian Ivec for help with the shape analysis, Josip Čačković for technical assistance, all members of Tolić and Pavin groups for discussions and advice, Ivana Šarić for the drawings. This work was funded by the European Research Council (ERC Consolidator Grant, GA number 647077), Croatian Science Foundation (Project IP-2014-09-4753), Croatian Science Foundation Cooperation Programme with Croatian Scientists in Diaspora "Research Cooperability" (Project PZS-2019-02-7653), and QuantiXLie Centre of Excellence, a project cofinanced by the Croatian Government and European Union through the European Regional Development Fund - the Competitiveness and Cohesion Operational Programme (Grant KK.01.1.1.01.0004). 


\section{Author Contribution}

M.M., I.K. and V.Š. performed the experiments, analyzed the data and assembled the figures. J.S. performed a lentivirus-based cell line construction. V.Š. and I.K. wrote the manuscript with input from M.M, J.S. and I.M.T. The experiments were conceived by J.S. and I.M.T. I.M.T. supervised the project.

\section{Competing Interests}

The authors declare no competing interest.

\section{Data Availability}

All data are available from the corresponding author upon request.

\section{Code Availability}

All scripts used in this study are available upon request. 


\section{Supplementary Tables}

Table S1. Spindle lengths, widths and diameters of the metaphase plate in control cells, HAUS6 and HAUS8 siRNA treated Hela cells stably expressing PRC1-GFP and RPE1 cells stably expressing CENP-A-GFP and Centrin1-GFP. All values are given as mean \pm SEM.

\begin{tabular}{|c|c|c|c|c|c|c|}
\hline & \multicolumn{3}{|c|}{ RPE1 cells stably expressing CENP- } & \multicolumn{3}{c|}{ HeLa cells stably expressing } \\
& \multicolumn{2}{|c|}{ A-GFP and Centrin1-GFP } & \multicolumn{3}{c|}{ PRC1-GFP } \\
\hline & Control & $\begin{array}{c}\text { HAUS6 } \\
\text { siRNA }\end{array}$ & $\begin{array}{c}\text { HAUS8 } \\
\text { siRNA }\end{array}$ & Control & $\begin{array}{c}\text { HAUS6 } \\
\text { siRNA }\end{array}$ & $\begin{array}{c}\text { HAUS8 } \\
\text { siRNA }\end{array}$ \\
\hline $\begin{array}{c}\text { Spindle length } \\
(\mu \mathrm{m})\end{array}$ & $13.5 \pm 0.3$ & $11.8 \pm 0.3$ & $11.7 \pm 0.2$ & $\begin{array}{c}11.3 \pm \\
0.2\end{array}$ & $\begin{array}{c}13.5 \pm \\
0.3\end{array}$ & $\begin{array}{c}11.9 \pm \\
0.2\end{array}$ \\
\hline $\begin{array}{c}\text { Spindle width } \\
(\mu \mathrm{m})\end{array}$ & $9.5 \pm 0.1$ & $13.0 \pm 0.3$ & $11.8 \pm 0.3$ & $\begin{array}{c}8.4 \pm \\
0.1\end{array}$ & $\begin{array}{c}10.2 \pm \\
0.3\end{array}$ & $\begin{array}{c}10.0 \pm \\
0.3\end{array}$ \\
\hline $\begin{array}{c}\text { Metaphase } \\
\text { plate diameter } \\
(\mu \mathrm{m})\end{array}$ & $9.5 \pm 0.1$ & $9.4 \pm 0.1$ & $9.3 \pm 0.2$ & $\begin{array}{c}13.6 \pm \\
0.2\end{array}$ & $\begin{array}{c}13.3 \pm \\
0.3\end{array}$ & $\begin{array}{c}13.1 \pm \\
0.2\end{array}$ \\
\hline
\end{tabular}




\section{Supplementary Figures}

A HeLa PRC1-GFP anti-HAUS6

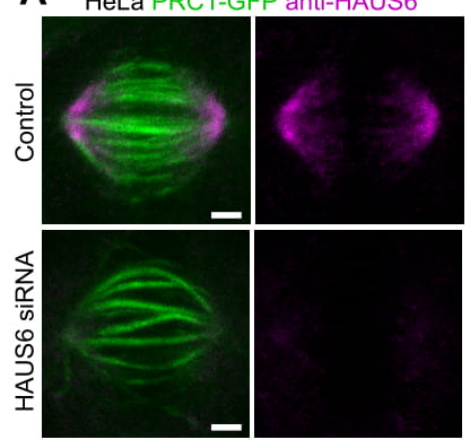

D RPE1 HAUS8 inducible

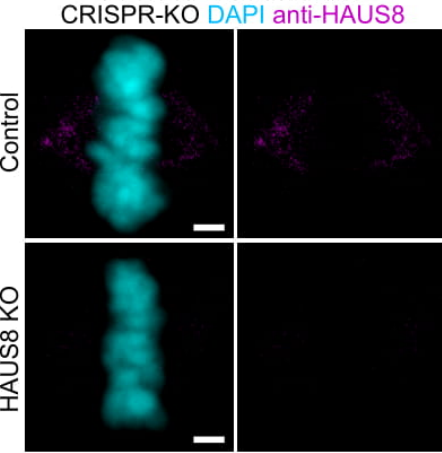

G

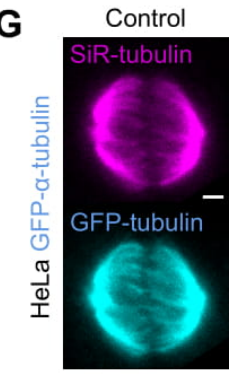

HAUS8 SiRNA

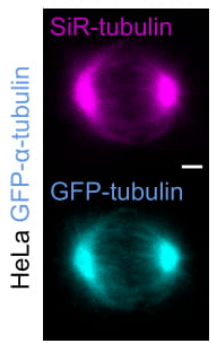

HeLa PRC1-GFP anti-HAUS8

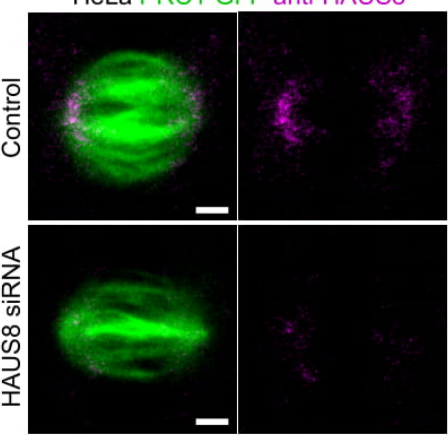

$\mathbf{F}$
B

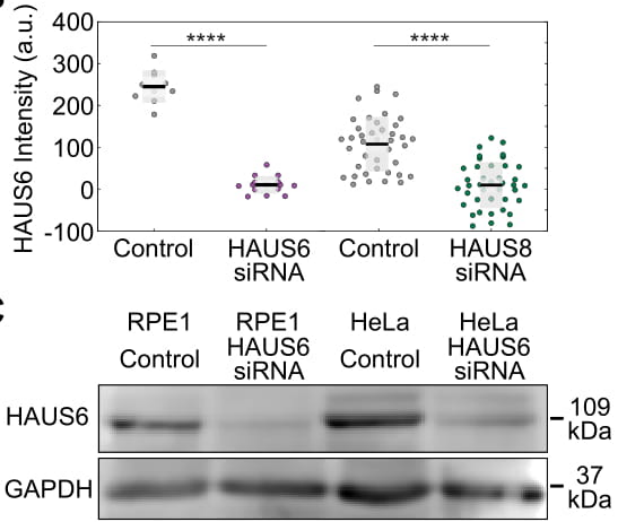
$\mathrm{kDa}$

4000
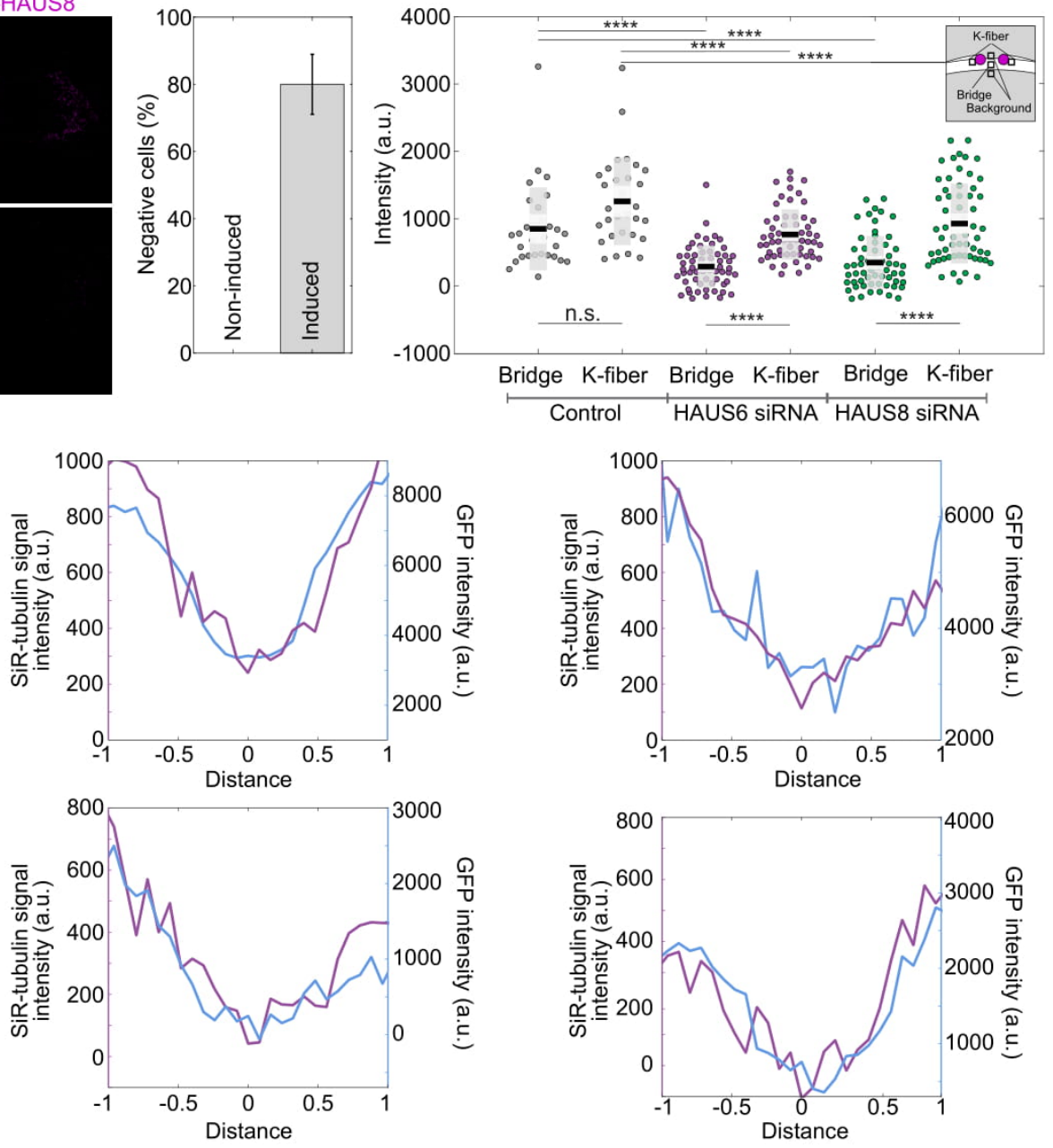

Supplementary Figure 1 (S1). (A) Fixed control, HAUS6 and HAUS8 siRNA-treated HeLa cells stably expressing PRC1-GFP (green) and immunostained for HAUS6 and HAUS8 (magenta). Chromosomes were stained with DAPI (not shown) to identify cells in metaphase. Images are sum intensity projections of nine central z-planes. (B) Univariate scatter plot of HAUS6 and HAUS8 intensity in control cells (gray) and cells depleted of HAUS6 (magenta) 
or HAUS8 (green). HAUS6 and HAUS8 intensity in treated cells is reduced for $96 \pm 2 \%$ and $82 \pm 16 \%$ compared to control cells, respectively. (C) Immunoblot analysis of HAUS6 siRNA treatment efficiency in RPE1 cells stably expressing CENP-A-GFP and Centrin1-GFP and HeLa cells stably expressing PRC1-GFP. HAUS6 antibody was used to validate the efficiency of knockdown, with GAPDH as the loading control. A representative image of three independent experiments is shown. (D) Fixed non-induced and induced RPE1 inducible CRISPR-Cas9 HAUS8 KO cells immunostained for HAUS8 (magenta) and $\alpha$-tubulin (not shown). Chromosomes were stained with DAPI (cyan) to identify cells in metaphase. Images are sum intensity projections of five central z-planes. (E) Percentage of HAUS8 negative cells (gray) determined by immunocytochemistry in relation to all DAPI-stained metaphase cells. In induced RPE1 inducible CRISPR/Cas9/HAUS8 cells, $80 \pm 9 \%$ of cells have no detectable HAUS8 signal. (F) Univariate scatter plot of the tubulin signal intensities of bridging and kfibers in control (gray) RPE1 cells stably expressing CENP-A-GFP and Centrin1-GFP and stained with SiR-tubulin, and upon HAUS6 (magenta) and HAUS8 (green) depletion. Schematic representation of the method for measuring the tubulin intensity of a bridging fiber and corresponding k-fibers (kinetochores are shown in magenta and tubulin signal in white). For the analysis, the average of two k-fibers is calculated and the average of background is subtracted from the tubulin intensity values of the bridging and k-fiber. Boxes represent standard deviation (dark gray), 95\% confidence interval of the mean (light gray) and mean value (black). (G) Intensity profiles of SiR-tubulin (magenta) and GFP-tubulin (cyan) signals in HeLa cells stably expressing GFP- $\alpha$-tubulin (cyan) and stained with SiR-tubulin (magenta). The values of two intensity profiles are adjusted to observe the level of overlapping between them and the minimum of the SiR-tubulin signal is set as a distance zero. All results were obtained from three independent experiments. Statistical analysis (B and F); t-test, p-value

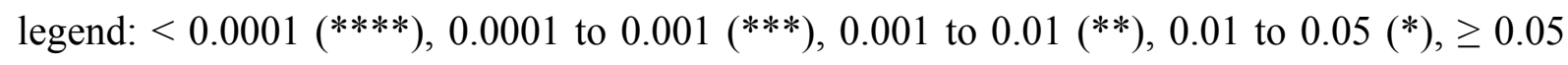
(ns). Scale bars, $2 \mu \mathrm{m}$. 

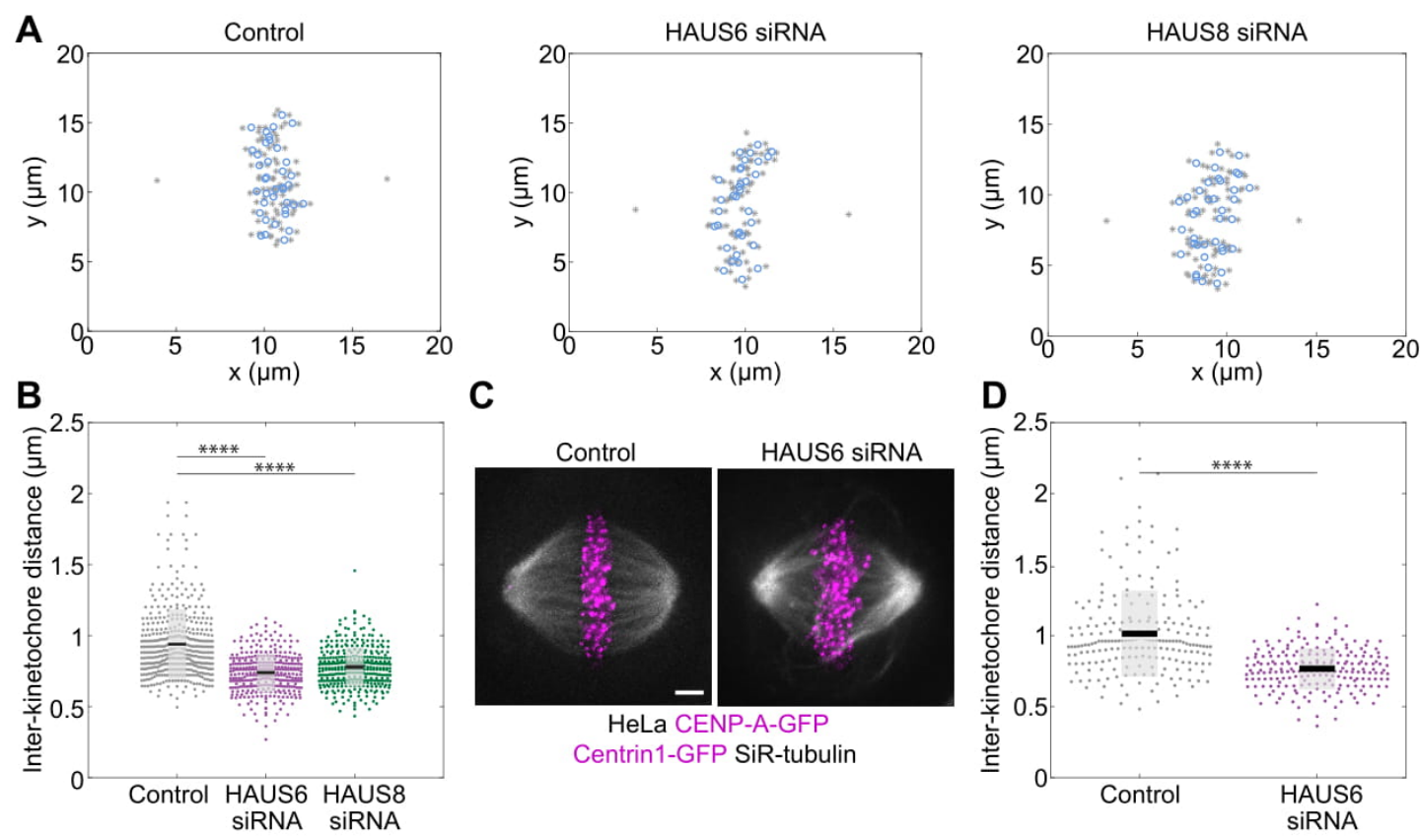

Supplementary Figure 2 (S2). (A) Coordinates of tracked kinetochore pairs (gray) in control (left) and in HAUS6 (middle) and HAUS8 (right) siRNA treated RPE1 cells stably expressing CENP-A-GFP and Centrin1-GFP. The sister kinetochore midpoint is shown as a blue circle and single asterisks denote positions of spindle poles and limits of the metaphase plate. (B) Univariate scatter plot of the interkinetochore distance in RPE1 cells stably expressing CENPA-GFP and Centrin1-GFP. The interkinetochore distance decreased from $0.99 \pm 0.04 \mu \mathrm{m}$ in control cells to $0.73 \pm 0.01 \mu \mathrm{m}$ and $0.78 \pm 0.01 \mu \mathrm{m}$ in HAUS6 and HAUS8 siRNA treated cells, respectively $(\mathrm{N}>400$ kinetochore pairs, $\mathrm{n}=10$ cells for each treatment). (C) HeLa cells stably expressing CENP-A-GFP and Centrin1-GFP (both in magenta) stained with SiRtubulin (gray) in control cells (left) and in cells following HAUS6 depletion (right). (D) Univariate scatter plot of the interkinetochore distance in HeLa cells stably expressing CENPA-GFP and Centrin1-GFP. The interkinetochore distance decreased from $1.01 \pm 0.02 \mu \mathrm{m}$ in control cells to $0.77 \pm 0.01 \mu \mathrm{m}$ following HAUS6 siRNA treatment $(\mathrm{N}>200$ kinetochore pairs, $\mathrm{n}=5$ cells). Boxes represent standard deviation (dark gray), 95\% confidence interval of the mean (light gray) and mean value (black). All results were obtained from three independent experiments. Statistical analysis (B and D); t-test, p-value legend: $<0.0001$ $(* * * *), 0.0001$ to $0.001\left(^{* * *}\right), 0.001$ to $0.01\left(^{* *}\right), 0.01$ to $0.05\left(^{*}\right), \geq 0.05$ (ns). Scale bars, 2 $\mu \mathrm{m}$. 

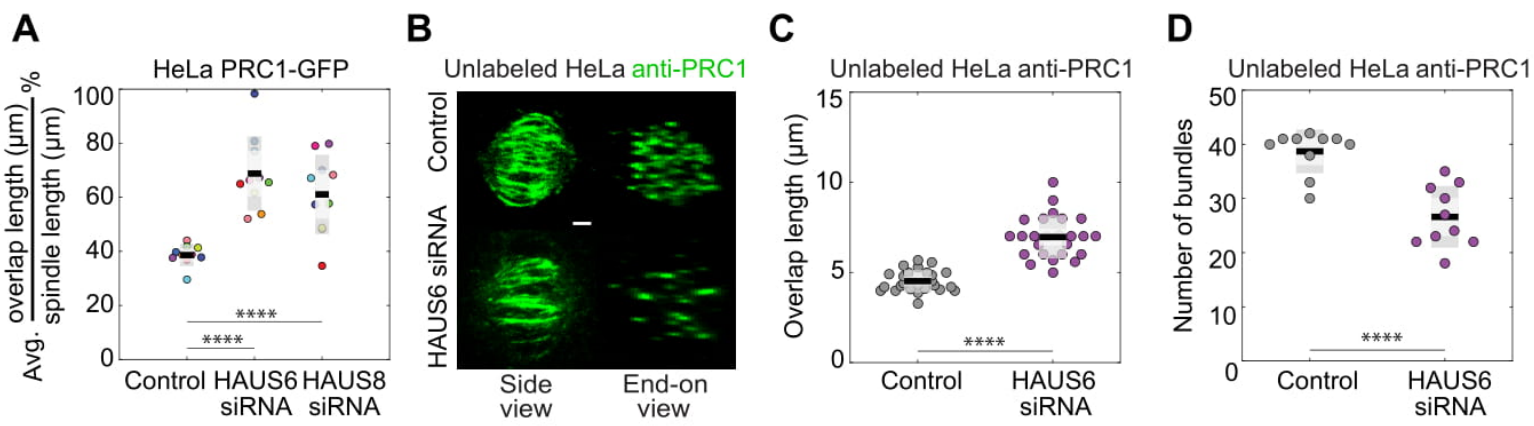

Supplementary Figure 3 (S3). (A) Univariate scatter plot of an average overlap length compared to spindle length in HeLa cell line stably expressing PRC1-GFP and stained with SiR-DNA. (B) Fixed control and HAUS6 siRNA treated unlabeled HeLa cells immunostained for PRC1 (green). Images are sum intensity projections of five central z-planes in a side view and sum projection of ten central z-planes in an end-on view. Corresponding overlap lengths (C) and number of bundles (D) shown in an univariate scatter plot. Boxes represent standard deviation (dark gray), 95\% confidence interval of the mean (light gray) and mean value (black). All results were obtained from three independent experiments. Statistical analysis; $t$ test, p-value legend: $<0.0001(* * * *), 0.0001$ to $0.001(* * *), 0.001$ to $0.01(* *), 0.01$ to 0.05 $(*), \geq 0.05(\mathrm{~ns})$. Scale bars, $2 \mu \mathrm{m}$. 
A
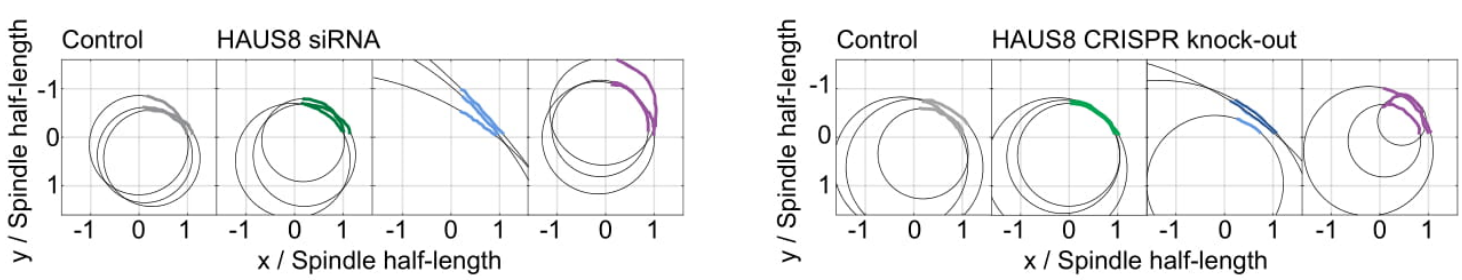

B

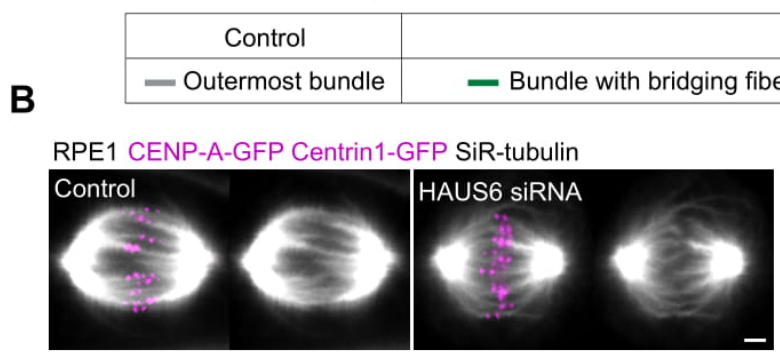

Augmin depletion

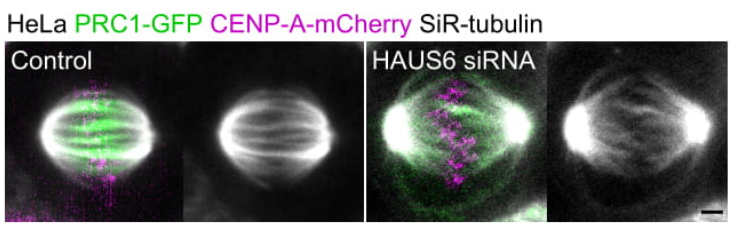

C
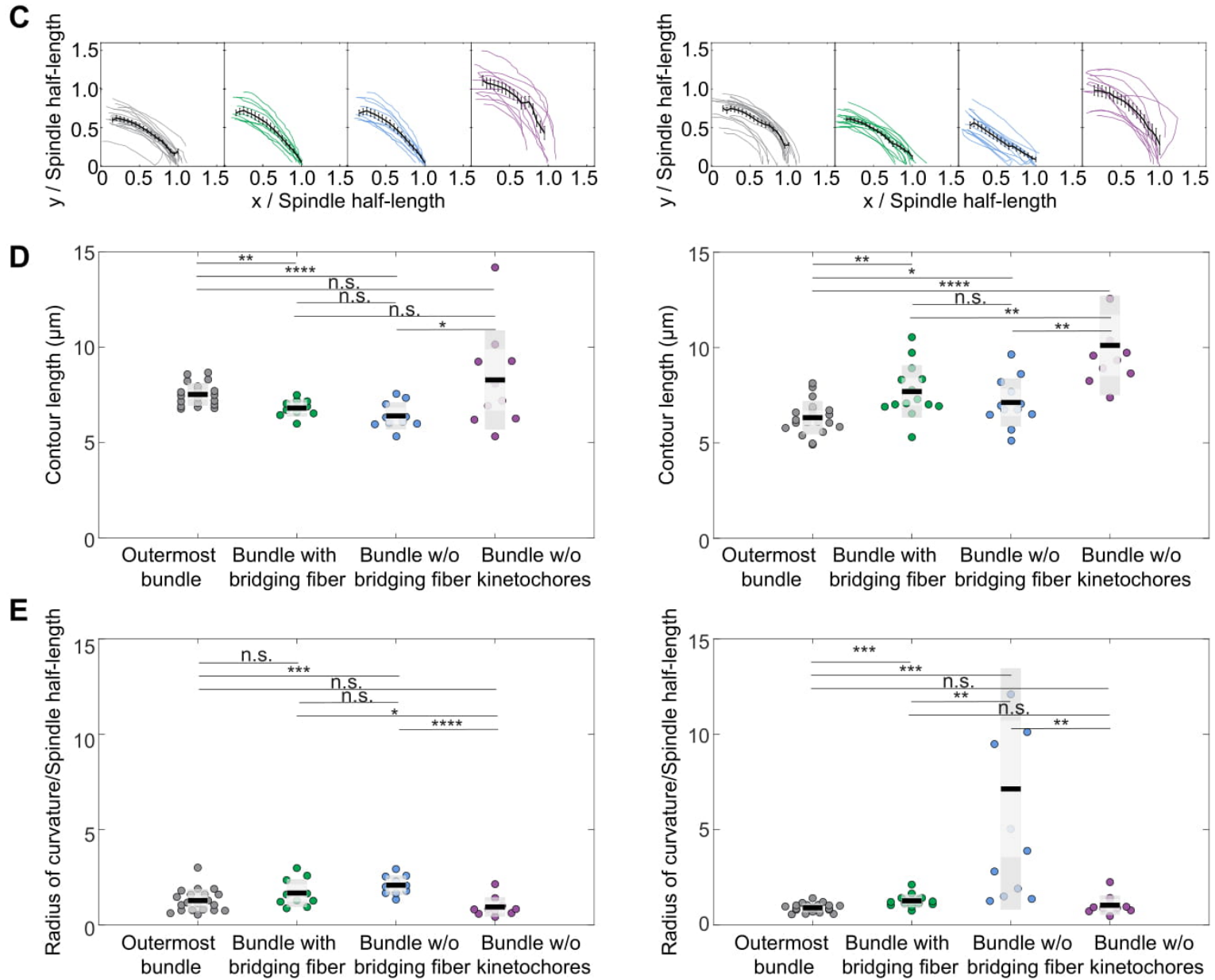

Supplementary Figure 4 (S4). (A) Examples of the circle-fitting analysis to determine the curvature of bundles in HAUS8 siRNA treated RPE1 cells stably expressing CENP-A-GFP and Centrin1-GFP (left) and RPE1 cells with induced CRISPR/Cas9/HAUS8 knock-out (right). Outermost bundle (gray) in non-induced cells and bundle with the bridging fiber (green), bundle without the bridging fiber (blue) and bundle without kinetochores (magenta) 
in induced cells. (B) Left panel - live images (maximum projections of 3 central z-planes) of metaphase spindles in control and HAUS6 siRNA treated SiR-tubulin (gray) stained RPE1 cells stably expressing CENP-A-GFP and Centrin1-GFP (both in magenta) and right panel live images (maximum projections of 3 central z-planes) of metaphase spindles in control and HAUS6 siRNA treated SiR-tubulin (gray) stained HeLa cells stably expressing PRC1-GFP (green) and CENP-A-mCherry (magenta). (C) Bundle contours in cells from (B); black lines show mean \pm SEM. (D) Univariate scatter plot of contour lengths and (E) radii of curvature. Boxes represent standard deviation (dark gray), 95\% confidence interval of the mean (light gray) and mean value (black). (C-E) Left panel shows results for RPE1 cells stably expressing CENP-A-GFP and Centrin1-GFP in control cells and in cells following HAUS6 depletion. Right panel shows results for HeLa cells stably expressing PRC1-GFP and CENP-A-mCherry in control cells and in cells following HAUS6 depletion. All results were obtained from three independent experiments. Statistical analysis; t-test, p-value legend: $<0.0001(* * * *), 0.0001$ to $0.001(* * *), 0.001$ to $0.01(* *), 0.01$ to $0.05(*), \geq 0.05$ (ns). Scale bars, $2 \mu \mathrm{m}$. 
Supplementary Video 1. SiR-tubulin (gray) stained RPE1 cells stably expressing CENP-AGFP and Centrin1-GFP (both in magenta) in 11 single Z-planes $(\Delta z=0.5 \mu \mathrm{m})$. The video shows control (left), and cells depleted of HAUS6 (middle) and HAUS8 (right). The video corresponds to still images from Fig. 1B. Scale bar, $2 \mu \mathrm{m}$.

Supplementary Video 2. Enlarged metaphase midzone of SiR-tubulin (gray) stained RPE1 cells stably expressing CENP-A-GFP and Centrin1-GFP (both in magenta). Video shows 11 single Z-planes $(\Delta \mathrm{z}=0.5 \mu \mathrm{m})$ of control (left), HAUS6 (middle) and HAUS8 (right) depleted cells. Scale bar, $2 \mu \mathrm{m}$

Supplementary Video 3. HeLa cells stably expressing PRC1-GFP (green) and stained with SiR-DNA (magenta) for chromosomes. Video shows the side view (left) of 21 single Z-planes $(\Delta \mathrm{z}=0.5 \mu \mathrm{m})$ in control (top), HAUS6 (middle) and HAUS8 (bottom) siRNA treated cells, as well as corresponding end-on views (right). Side view spindles are smoothed with 0.5 pixelsigma Gaussian blur. Scale bar, $2 \mu \mathrm{m}$.

Supplementary Video 4. RPE1 cells with induced CRISPR/Cas9/HAUS8 knock-out stained with SiR-tubulin (gray) and Hoechst dye (magenta) for chromosomes. Video shows 9 single Z planes $(\Delta \mathrm{z}=0.5 \mu \mathrm{m})$. Scale bar, $2 \mu \mathrm{m}$.

Supplementary Video 5. Time lapse video of control (left) and HAUS6-depleted (right) RPE1 cells stably expressing PA- $\alpha$-tubulin and stained with SiR-tubulin (magenta) before and after the photoactivation (white). Time, seconds. Scale bar, $2 \mu \mathrm{m}$. 


\section{References}

Brust-Mascher, I., Sommi, P., Cheerambathur, D. K., and Scholey, J. M. (2009). Kinesin-5-dependent poleward flux and spindle length control in Drosophila embryo mitosis. Mol Biol Cell, 20(6), 1749-1762. doi:10.1091/mbc.E08-10-1033

Buda, R., Vukusic, K., and Tolic, I. M. (2017). Dissection and characterization of microtubule bundles in the mitotic spindle using femtosecond laser ablation. Methods Cell Biol, 139, 81-101. doi:10.1016/bs.mcb.2016.11.007

David, A. F., Roudot, P., Legant, W. R., Betzig, E., Danuser, G., and Gerlich, D. W. (2019). Augmin accumulation on long-lived microtubules drives amplification and kinetochore-directed growth. The Journal of Cell Biology, 218(7), 2150-2168. doi:10.1083/jcb.201805044

Dudka, D., Noatynska, A., Smith, C. A., Liaudet, N., McAinsh, A. D., and Meraldi, P. (2018). Complete microtubule-kinetochore occupancy favours the segregation of merotelic attachments. Nature Communications, 9. doi:10.1038/s41467-018-04427-x

Elting, M. W., Prakash, M., Udy, D. B., and Dumont, S. (2017). Mapping Load-Bearing in the Mammalian Spindle Reveals Local Kinetochore Fiber Anchorage that Provides Mechanical Isolation and Redundancy. Current Biology, 27(14), 2112-2122.e2115. doi:10.1016/j.cub.2017.06.018

Ganem, N. J., Upton, K., and Compton, D. A. (2005). Efficient mitosis in human cells lacking poleward microtubule flux. Current Biology, 15(20), 1827-1832. doi:10.1016/j.cub.2005.08.065

Goshima, G., Mayer, M., Zhang, N., Stuurman, N., and Vale, R. D. (2008). Augmin: a protein complex required for centrosome-independent microtubule generation within the spindle. The Journal of Cell Biology, 181(3), 421-429. doi:10.1083/jcb.200711053

Goshima, G., Wollman, R., Goodwin, S. S., Zhang, N., Scholey, J. M., Vale, R. D., and Stuurman, N. (2007). Genes required for mitotic spindle assembly in Drosophila S2 cells. Science, 316(5823), 417-421. doi:10.1126/science.1141314

Hayward, D., Metz, J., Pellacani, C., and Wakefield, J. G. (2014). Synergy between multiple microtubulegenerating pathways confers robustness to centrosome-driven mitotic spindle formation. Developmental cell, 28(1), 81-93. doi:10.1016/j.devcel.2013.12.001

Jagrić, M., Risteski, P., Jelena, M., Milas, A., and Tolić, I. M. (2020). Optogenetic control of PRC1 reveals that bridging fibers promote chromosome alignment by overlap length-dependent forces. bioRxiv.

Kajtez, J., Solomatina, A., Novak, M., Polak, B., Vukušić, K., Rüdiger, J., Cojoc, G., Milas, A., Šumanovac Šestak, I., Risteski, P., Tavano, F., Klemm, A. H., Roscioli, E., Welburn, J., Cimini, D., Glunčić, M., Pavin, N., and Tolić, I. M. (2016). Overlap microtubules link sister k-fibres and balance the forces on bi-oriented kinetochores. Nature Communications, 7(1), 10298. doi:10.1038/ncomms10298

Kamasaki, T., O'Toole, E., Kita, S., Osumi, M., Usukura, J., McIntosh, J. R., and Goshima, G. (2013). Augmindependent microtubule nucleation at microtubule walls in the spindle. The Journal of Cell Biology, 202(1), 25-33. doi:10.1083/jcb.201304031

Kanatani, K., and Rangarajan, P. (2011). Hyper least squares fitting of circles and ellipses. Computational Statistics \& Data Analysis, 55(6), 2197-2208. doi:10.1016/j.csda.2010.12.012

Kirschner, and Mitchison. (1986). Beyond self-assembly: from microtubules to morphogenesis. Cell, 45(3), 329342. doi:10.1016/0092-8674(86)90318-1

Lampson, M. A., and Grishchuk, E. L. (2017). Mechanisms to Avoid and Correct Erroneous KinetochoreMicrotubule Attachments. Biology-Basel, 6(1). doi:10.3390/biology6010001

Lawo, S., Bashkurov, M., Mullin, M., Ferreria, M. G., Kittler, R., Habermann, B., Tagliaferro, A., Poser, I., Hutchins, J. R., Hegemann, B., Pinchev, D., Buchholz, F., Peters, J. M., Hyman, A. A., Gingras, A. C., and Pelletier, L. (2009). HAUS, the 8-subunit human Augmin complex, regulates centrosome and spindle integrity. Curr Biol, 19(10), 816-826. doi:10.1016/j.cub.2009.04.033

Li, J., Dallmayer, M., Kirchner, T., Musa, J., and Grunewald, T. G. P. (2018). PRC1: Linking Cytokinesis, Chromosomal Instability, and Cancer Evolution. Trends Cancer, 4(1), 59-73. doi:10.1016/j.trecan.2017.11.002

Mastronarde, D. N., McDonald, K. L., Ding, R., and Mclntosh, J. R. (1993). Interpolar spindle microtubules in PTK cells. The Journal of Cell Biology, 123(6), 1475-1489. doi:10.1083/jcb.123.6.1475

McDonald, K. L., O'Toole, E. T., Mastronarde, D. N., and McIntosh, J. R. (1992). Kinetochore microtubules in PTK cells. The Journal of Cell Biology, 118(2), 369-383. doi:10.1083/jcb.118.2.369

Mclntosh, J. R. (2016). Mitosis. Cold Spring Harbor Perspectives in Biology, 8(9). doi:10.1101/cshperspect.a023218 
bioRxiv preprint doi: https://doi.org/10.1101/2020.09.10.291740; this version posted November 19, 2020. The copyright holder for this preprint (which was not certified by peer review) is the author/funder, who has granted bioRxiv a license to display the preprint in perpetuity. It is made available under aCC-BY-NC-ND 4.0 International license.

Mitchison, T. J. (2005). Mechanism and function of poleward flux in Xenopus extract meiotic spindles. Philos Trans R Soc Lond B Biol Sci, 360(1455), 623-629. doi:10.1098/rstb.2004.1616

Miyamoto, D. T., Perlman, Z. E., Burbank, K. S., Groen, A. C., and Mitchison, T. J. (2004). The kinesin Eg5 drives poleward microtubule flux in Xenopus laevis egg extract spindles. J Cell Biol, 167(5), 813-818. doi:10.1083/jcb.200407126

Mollinari, C., Kleman, J. P., Jiang, W., Schoehn, G., Hunter, T., and Margolis, R. L. (2002). PRC1 is a microtubule binding and bundling protein essential to maintain the mitotic spindle midzone. J Cell Biol, 157(7), 1175-1186. doi:10.1083/jcb.200111052

Musacchio, A., and Salmon, E. D. (2007). The spindle-assembly checkpoint in space and time. Nature Reviews Molecular Cell Biology, 8(5), 379-393. doi:10.1038/nrm2163

Nicklas, R. B., Ward, S. C., and Gorbsky, G. J. (1995). Kinetochore Chemistry Is Sensitive to Tension and May Link Mitotic Forces to a Cell-Cycle Checkpoint. Journal of Cell Biology, 130(4), 929-939. doi:10.1083/jcb.130.4.929

Novak, M., Polak, B., Simunić, J., Boban, Z., Kuzmić, B., Thomae, A. W., Tolić, I. M., and Pavin, N. (2018). The mitotic spindle is chiral due to torques within microtubule bundles. Nature Communications, 9(1), 3571. doi:10.1038/s41467-018-06005-7

O'Toole, E., Morphew, M., and McIntosh, J. R. (2020). Electron tomography reveals aspects of spindle structure important for mechanical stability at metaphase. Mol Biol Cell, 31(3), 184-195. doi:10.1091/mbc.E1907-0405

Pavin, N., and Tolić, I. M. (2016). Self-Organization and Forces in the Mitotic Spindle. Annual Review of Biophysics, 45(1), 279-298. doi:10.1146/annurev-biophys-062215-010934

Pereira, A. J., and Maiato, H. (2010). Improved kymography tools and its applications to mitosis. Methods, 51(2), 214-219. doi:10.1016/j.ymeth.2010.01.016

Petry, S. (2016). Mechanisms of Mitotic Spindle Assembly. Annu Rev Biochem, 85, 659-683. doi:10.1146/annurev-biochem-060815-014528

Petry, S., Groen, A. C., Ishihara, K., Mitchison, T. J., and Vale, R. D. (2013). Branching microtubule nucleation in Xenopus egg extracts mediated by augmin and TPX2. Cell, 152(4), 768-777. doi:10.1016/j.cell.2012.12.044

Petry, S., Pugieux, C., Nedelec, F. J., and Vale, R. D. (2011). Augmin promotes meiotic spindle formation and bipolarity in Xenopus egg extracts. Proceedings of the National Academy of Sciences of the United States of America, 108(35), 14473-14478. doi:10.1073/pnas.1110412108

Polak, B., Risteski, P., Lesjak, S., and Tolić, I. M. (2017). PRC-1 labeled microtubule bundles and kinetochore pairs show one-to-one association in metaphase. EMBO Reports, 18(2), 217-230. doi:10.15252/embr.201642650

Prosser, S. L., and Pelletier, L. (2017). Mitotic spindle assembly in animal cells: a fine balancing act. Nature Reviews Molecular Cell Biology, 18(3), 187-201. doi:10.1038/nrm.2016.162

Simunić, J., and Tolić, I. M. (2016). Mitotic Spindle Assembly: Building the Bridge between Sister K-Fibers. Trends in Biochemical Sciences, 41(10), 824-833. doi:10.1016/j.tibs.2016.07.004

Song, J.-G., King, M. R., Zhang, R., Kadzik, R. S., Thawani, A., and Petry, S. (2018). Mechanism of how augmin directly targets the $\mathrm{y}$-tubulin ring complex to microtubules. Journal of Cell Biology, 217(7), 2417-2428. doi:10.1083/jcb.201711090

Steblyanko, Y., Rajendraprasad, G., Osswald, M., Eibes, S., Jacome, A., Geley, S., Pereira, A. J., Maiato, H., and Barisic, M. (2020). Microtubule poleward flux in human cells is driven by the coordinated action of four kinesins. The EMBO Journal, e105432. doi:10.15252/embj.2020105432

Suresh, P., Long, A. F., and Dumont, S. (2020). Microneedle manipulation of the mammalian spindle reveals specialized, short-lived reinforcement near chromosomes. eLife, 9. doi:10.7554/eLife.53807

Taylor, S. S., Ha, E., and McKeon, F. (1998). The human homologue of Bub3 is required for kinetochore localization of Bub1 and a Mad3/Bub1-related protein kinase. Journal of Cell Biology, 142(1), 1-11. doi:10.1083/jcb.142.1.1

Tolic, I. M. (2018). Mitotic spindle: kinetochore fibers hold on tight to interpolar bundles. Eur Biophys J, 47(3), 191-203. doi:10.1007/s00249-017-1244-4

Tolic, I. M., and Pavin, N. (2016). Bridging the gap between sister kinetochores. Cell Cycle, 15(9), 1169-1170. doi:10.1080/15384101.2016.1157976

Uehara, R., and Goshima, G. (2010). Functional central spindle assembly requires de novo microtubule generation in the interchromosomal region during anaphase. J Cell Biol, 191(2), 259-267. doi:10.1083/jcb.201004150 
bioRxiv preprint doi: https://doi org/10.1101/2020.0910.291740; this version posted November 19, 2020. The copyright holder for this preprint (which was not certified by peer review) is the author/funder, who has granted bioRxiv a license to display the preprint in perpetuity. It is made available under aCC-BY-NC-ND 4.0 International license.

Uehara, R., Kamasaki, T., Hiruma, S., Poser, I., Yoda, K., Yajima, J., Gerlich, D. W., and Goshima, G. (2016). Augmin shapes the anaphase spindle for efficient cytokinetic furrow ingression and abscission. Mol Biol Cell, 27(5), 812-827. doi:10.1091/mbc.E15-02-0101

Uehara, R., Nozawa, R.-s., Tomioka, A., Petry, S., Vale, R. D., Obuse, C., and Goshima, G. (2009). The augmin complex plays a critical role in spindle microtubule generation for mitotic progression and cytokinesis in human cells. Proceedings of the National Academy of Sciences, 106(17), 6998-7003. doi:10.1073/pnas.0901587106

Verma, V., and Maresca, T. J. (2019). Direct observation of branching MT nucleation in living animal cells. The Journal of Cell Biology, 218(9), 2829-2840. doi:10.1083/jcb.201904114

Vukušić, K., Buđa, R., Bosilj, A., Milas, A., Pavin, N., and Tolić, I. M. (2017). Microtubule Sliding within the Bridging Fiber Pushes Kinetochore Fibers Apart to Segregate Chromosomes. Developmental Cell, 43(1), 11-23.e16. doi:10.1016/j.devcel.2017.09.010

Waters, J. C., Skibbens, R. V., and Salmon, E. D. (1996). Oscillating mitotic newt lung cell kinetochores are, on average, under tension and rarely push. Journal of Cell Science, 109, 2823-2831.

Wu, G., Lin, Y., Wei, R., Chen, Y., Shan, Z., and Lee, W. (2008). Hice1, a novel microtubule-associated protein required for maintenance of spindle integrity and chromosomal stability in human cells. Molecular and Cellular Biology, 28(11), 3652-3662. doi:10.1128/MCB.01923-07

Zhu, H., Coppinger, J. A., Jang, C.-Y., Yates, J. R., and Fang, G. (2008). FAM29A promotes microtubule amplification via recruitment of the NEDD1-gamma-tubulin complex to the mitotic spindle. The Journal of Cell Biology, 183(5), 835-848. doi:10.1083/jcb.200807046 